SANDIA REPORT

SAND2006-2785

Unlimited Release

Printed June 2006
Technical Librarv

MS 0899

4536

\title{
Verification Test Problems for the Calculation of Probability of Loss of Assured Safety in Temperature- Dependent Systems with Multiple Weak and Strong Links
}

J.C. Helton, J.D. Johnson, W.L. Oberkampf

Prepared by

Sandia National Laboratories

Albuquerque, New Mexico 87185 and Livermore, California 94550

Sandia is a multiprogram laboratory operated by Sandia Corporation, a Lockheed Martin Company, for the United States Department of Energy's National Nuclear Security Administration under Contract DE-AC04-94AL85000.

Approved for public release; further dissemination unlimited.

\section{Sandia National Laboratories}


Issued by Sandia National Laboratories, operated for the United States Department of Energy by Sandia Corporation.

NOTICE: This report was prepared as an account of work sponsored by an agency of the United States Government. Neither the United States Government, nor any agency thereof, nor any of their employees, nor any of their contractors, subcontractors, or their employees, make any warranty, express or implied, or assume any legal liability or responsibility for the accuracy, completeness, or usefulness of any information, apparatus, product, or process disclosed, or represent that its use would not infringe privately owned rights. Reference herein to any specific commercial product, process, or service by trade name, trademark, manufacturer, or otherwise, does not necessarily constitute or imply its endorsement, recommendation, or favoring by the United States Government, any agency thereof, or any of their contractors or subcontractors. The views and opinions expressed herein do not necessarily state or reflect those of the United States Government, any agency thereof, or any of their contractors.

Printed in the United States of America. This report has been reproduced directly from the best available copy.

Available to DOE and DOE contractors from

U.S. Department of Energy

Office of Scientific and Technical Information

P.O. Box 62

Oak Ridge, TN 37831

Telephone: (865)576-8401

Facsimile: (865)576-5728

E-Mail: reports@adonis.osti.gov

Online ordering: http://www.doc.gov/bridge

Available to the public from

U.S. Department of Commerce

National Technical Information Service

5285 Port Royal Rd.

Springfield, VA 22161

Telephone: (800)553-6847

Facsimile: (703)605-6900

E-Mail: orders@ntis.fedworld.gov

Online ordering: http://www.ntis.gov/ordering.htm

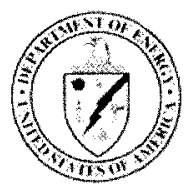


SAND2006-2785

Unlimited Release

Printed June 2006

\title{
Verification Test Problems for the Calculation of Probability of Loss of Assured Safety in Temperature- Dependent Systems with Multiple Weak and Strong Links
}

\author{
J.C. Helton, ${ }^{a}$ J.D. Johnson, ${ }^{b}$ and W.L. Oberkamp ${ }^{\mathrm{c}}$ \\ aDepartment of Mathematics and Statistics, Arizona State University, Tempe, AZ 85287-1804 USA \\ bProStat, Mesa, AZ 85204-5326 USA \\ 'Sandia National Laboratories, Albuquerque, NM 87185-0776 USA
}

\begin{abstract}
Four verification test problems are presented for checking the conceptual development and computational implementation of calculations to determine the probability of loss of assured safety (PLOAS) in temperaturedependent systems with multiple weak links (WLs) and strong links (SLs). The problems are designed to test results obtained with the following definitions of loss of assured safety: (i) Failure of all SLs before failure of any WL, (ii) Failure of any SL before failure of any WL, (iii) Failure of all SLs before failure of all WLs, and (iv) Failure of any SL before failure of all WLs. The test problems are based on assuming the same failure properties for all links, which results in problems that have the desirable properties of fully exercising the numerical integration procedures required in the evaluation of PLOAS and also possessing simple algebraic representations for PLOAS that can be used for verification of the analysis.
\end{abstract}

Key Words: Aleatory uncertainty, Competing risk, Epistemic uncertainty, Fire environment, High consequence system, Probability of loss of assured safety, Reliability, Strong link, Verification, Weak link 


\section{Acknowledgments}

Review at Sandia National Laboratories provided by T. Brown and C. Sallaberry. Editorial support provided by F. Puffer and J. Ripple of Tech Reps, a division of Ktech Corporation. 


\section{Contents}

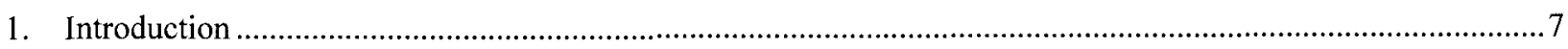

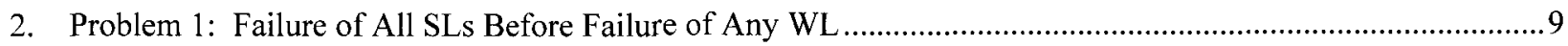

3. Problem 2: Failure of Any SL Before Failure of Any WL ......................................................................... 17

4. Problem 3: Failure of All SLs Before Failure of All WLs ........................................................................23

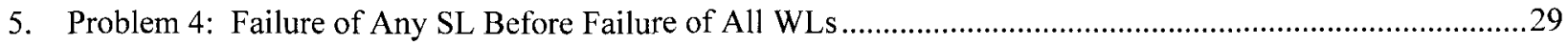

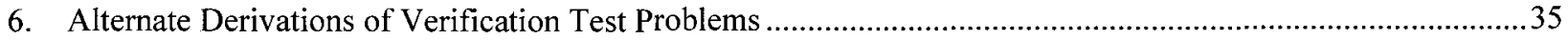

6.1 Problem 1: Failure of All SLs before Failure of Any WL ...................................................................

6.2 Problem 2: Failure of Any SL before Failure of Any WL ....................................................................36

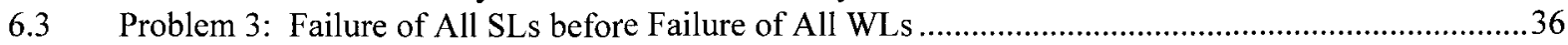

6.4 Problem 4: Failure of Any SL before Failure of All WLs................................................................37

6.5 Derivation of Verification Test Problems Based on Hypergeometric Probability Distribution ..............37

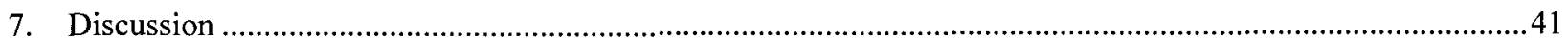

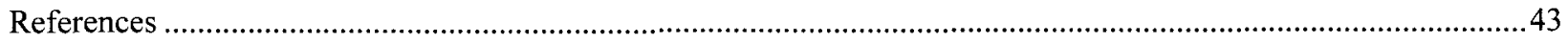




\section{Tables}

Table 1. Values for PLOAS (i.e., $\overline{p F}(\infty)$ in Eq. (2.5)) for Different Numbers of WLs and SLs Predicated on the Assumptions that (i) Loss of Assured Safety Corresponds to Failure of All SLs Before Failure of Any WL, (ii) The Failures of the Individual Links are Independent, and (iii) All Links Have the Same Distribution for Failure Time

Table 2. Representation of Value $p F$ for PLOAS Under Fire Conditions for WL/SL System With $n W L$ WLs, $n S L$ SLs, and the Assumptions that (i) a Link Fails Instantly When it Reaches its Failure Temperature and (ii) Loss of Assured Safety Corresponds to Failure of all SLs Before Failure of Any WL

Table 3. Deviations from Decimal Results in Table 1 Observed in Determination of PLOAS with SamplingBased Procedures and Samples of Size $10^{6}$

Table 4. Values for PLOAS (i.e., $\overline{p F}(\infty)$ in Eq. (3.4)) for Different Numbers of WLs and SLs Predicated on the Assumptions that (i) Loss of Assured Safety Corresponds to Failure of Any SL Before Failure of Any WL, (ii) the Failures of the Individual Links are Independent, and (iii) All Links Have the Same Distribution for Failure Time.

Table 5. Representation of Value $p F$ for PLOAS Under Fire Conditions for WL/SL System With $n W L$ WLs, $n S L$ SLs, and the Assumptions that (i) a Link Fails Instantly When it Reaches its Failure Temperature and (ii) Loss of Assured Safety Corresponds to Failure of Any SL Before Failure of Any WL

Table 6. Deviations from Decimal Results in Table 4 Observed in Determination of PLOAS with SamplingBased Procedures and Samples of Size $10^{6}$

Table 7. Values for PLOAS (i.e., $\overline{p F}(\infty)$ in Eq. (4.4)) for Different Numbers of WLs and SLs Predicated on the Assumptions that (i) Loss of Assured Safety Corresponds to Failure of All SLs Before Failure of All WLs, (ii) the Failures of the Individual Links are Independent, and (iii) All Links Have the Distribution for Failure Time

Table 8. Representation of Value $p F$ for PLOAS Under Fire Conditions for WL/SL System With $n W L$ WLs, $n S L$ SLs, and the Assumptions that (i) a Link Fails Instantly When it Reaches its Failure Temperature and (ii) Loss of Assured Safety Corresponds to Failure of All SLs Before Failure of All WL

Table 9. Deviations from Decimal Results in Table 7 Observed in Determination of PLOAS with SamplingBased Procedures and Samples of Size $10^{6}$

Table 10. Values for PLOAS (i.e., $\overline{p F}(\infty)$ in Eq. (5.4)) for Different Numbers of WLs and SLs Predicated on the Assumptions that (i) Loss of Assured Safety Corresponds to Failure of Any SL Before Failure of All WLs, (ii) the Failures of the Individual Links are Independent, and (iii) All Links Have the Same Distribution for Failure Time

Table 11. Representation of Value $p F$ for PLOAS Under Fire Conditions for WL/SL System With $n W L$ WLs, $n S L$ SLs, and the Assumptions that (i) a Link Fails Instantly When it Reaches its Failure Temperature and (ii) Loss of Assured Safety Corresponds to Failure of Any SL Before Failure of all WLs.....

Table 12. Deviations from Decimal Results in Table 10 Observed in Determination of PLOAS with SamplingBased Procedures and Samples of Size $10^{6}$ 


\section{Introduction}

Analysis verification is an essential part of an analysis of any complex system. ${ }^{1-10}$ One component of analysis verification is the use of verification test problems that have known solutions and possess the property of extensively exercising the software that implements the analysis under consideration. Then, deviations from the known solution indicate errors in the implementation of the analysis, and agreement with the known solution provides an indication, but not an absolute proof, that the analysis is implemented correctly.

A previous presentation illustrates the use of a simple verification test problem in the comparison of two approaches to the calculation of probability of loss of assured safety (PLOAS) for a temperature-dependent system with a single weak link (WL) and multiple strong links (SLs) (see Sect. 7, Ref. [11]). This test problem was very helpful in identifying the existence of an error in one of the approaches.

The indicated test problem is specific to a WL/SL system with one WL and multiple SLs and is based on the assignment of the same properties to all links (see Eq. (7.4.1), Ref. [11]). The result is a test problem that thoroughly exercises the numerical procedures used in the calculation of PLOAS while, at the same time, having a simple known solution.

The purpose of this presentation is to develop verification test problems for temperature-dependent systems with multiple weak and strong links through use of the indicated idea of assigning the same properties to all links. Further, the test problems are developed for several different definitions of loss of assured safety, with each of these definitions corresponding to a different time-dependent pattern of WL/SL failures. The determination of PLOAS for WL/SL systems falls in the general area of study known as competing risk analysis or, equivalently, competing failure analysis. ${ }^{12-16}$ 
This page intentionally left blank. 


\section{Problem 1: Failure of All SLs Before Failure of Any WL}

Verification Test Problem 1 is predicated on the assumption that loss of assured safety corresponds to all SLs failing before any WL fails. With this assumption, and the additional assumption that the failures of the individual links are independent, PLOAS as a function of time is given by

$$
\overline{p F}(t)=\text { probability that all SLs fail before time } t \text { and all WLs fail after time } t
$$

$$
\begin{aligned}
& =\sum_{k=1}^{n S L}\left(\int_{0}^{t}\left\{\prod_{\substack{l=1 \\
l \neq k}}^{n S L} C D F_{S L, l}(\tau)\right\}\left\{\prod_{j=1}^{n W L}\left[1-C D F_{W L, j}(\tau)\right]\right\} \mathrm{d} C D F_{S L, k}(\tau)\right) \\
& =\sum_{k=1}^{n S L}\left(\int_{0}^{t}\left\{\prod_{\substack{l=1 \\
l \neq k}}^{n S L} C D F_{S L, l}(\tau)\right\}\left\{\prod_{j=1}^{n W L}\left[1-C D F_{W L, j}(\tau)\right]\right\}\left\{\mathrm{d} C D F_{S L, k}(\tau) / \mathrm{d} \tau\right\} \mathrm{d} \tau\right),
\end{aligned}
$$

where the first integral is a Riemann-Stieltjes integral (i.e., an integral of the form $\int_{a}^{b} f(t) \mathrm{d} g(t)$; see Sect. 2.9, Ref. [16]), the second integral is the corresponding Riemann integral (i.e., an integral of the form $\int_{a}^{b} f(t) g^{\prime}(t) \mathrm{d} t$; see Theorem 29.8, p. 200, Ref. [16]), and $C D F_{W L, j}(\tau)$ and $C D F_{S L, k}(\tau)$ are defined by

$C D F_{W L, j}(\tau)=$ probability that $\mathrm{WL} j$ fails between time 0 and time $\tau$

and

$C D F_{S L, k}(\tau)=$ probability that SL $k$ fails between time 0 and time $\tau$,

respectively. As an example, the functions $C D F_{W L, j}$ and $C D F_{S L, k}$ can be derived as described in Sect. 7.1 of Ref. [11].

The notation $\overline{p F}(t)$ is retained from Sect. 7.1 of Ref. [11] because, depending on the definition of $C D F_{W L, j}$ and $C D F_{S L, k}, \overline{p F}(t)$ could be an expected value over epistemic uncertainty or possibly over both epistemic uncertainty and aleatory uncertainty. As a reminder, epistemic uncertainty derives from a lack of knowledge about a quantity that is assumed to have a fixed value in the context of the particular analysis under consideration. The complementary concept is aleatory uncertainty, which is an uncertainty that derives from an inherent randomness in the values that a quantity can take on. ${ }^{17-25}$

The integrals defining $\overline{p F}(t)$ in Eq. (2.1) are obtained from the approximations 


$$
\begin{aligned}
\overline{p F}(t) \cong \sum_{k=1}^{n S L}\left(\sum_{i=1}^{n}\left\{\prod_{\substack{l=1 \\
l \neq k}}^{n S L} C D F_{S L, l}\left(\tau_{i-1}\right)\right\}_{1}\left\{\prod_{j=1}^{n W L}\left[1-C D F_{W L, j}\left(\tau_{i}\right)\right]\right\}_{2}\left\{C D F_{S L, k}\left(\tau_{i}\right)-C D F_{S L, k}\left(\tau_{i-1}\right)\right\}_{3}\right) \\
=\sum_{k=1}^{n S L}\left(\sum_{i=1}^{n}\left\{\prod_{\substack{l=1 \\
l \neq k}}^{n S L} C D F_{S L, l}\left(\tau_{i-1}\right)\right\}\left\{\prod_{1}^{n W L}\left[1-C D F_{W L, j}\left(\tau_{i}\right)\right]\right\}_{2}\left\{\frac{C D F_{S L, k}\left(\tau_{i}\right)-C D F_{S L, k}\left(\tau_{i-1}\right)}{\Delta \tau_{i}}\right\}_{4} \Delta \tau_{i}\right)
\end{aligned}
$$

for $0=\tau_{0}<\tau_{1}<\ldots<\tau_{n}=t$ and $\Delta \tau_{i}=\tau_{\mathrm{i}}-\tau_{i-1}$. Specifically, $\{\sim\}_{1}$ is the probability that all SLs except SL $k$ fail before time $\tau_{i-1} ;\{\sim\}_{2}$ is the probability that all WLs fail after time $\tau_{i} ;\{\sim\}_{3}$ is the probability that SL $k$ fails between $\tau_{i-1}$ and $\tau_{i}$; and $\{\sim\}_{4}$ is an approximation to $\mathrm{d} C D F_{S L, k}(\tau) / \mathrm{d} \tau$. The integrals in Eq. (2.1) then result in the limit as $\Delta \tau_{i}$ goes to zero.

As already indicated, the failure times for the links are assumed to be independent. This assumption is unlikely to be satisfied in a real analysis as the links would be experiencing heating in the same fire. However, the objective is to derive a verification test problem rather than a model of high conceptual fidelity.

The desired verification test results by assuming all links have the same distribution for failure time. With this assumption, there exists a function $p(\tau)$ such that

$$
p(\tau)=C D F_{W L, j}(\tau)=C D F_{S L, k}(\tau)
$$

for $j=1,2, \ldots, n W L, k=1,2, \ldots, n S L$, and $0 \leq \tau$. Further, it is also assumed that the problem starts at time $0, p(0)=$ 0 , and $p(\infty)=1$.

With the indicated assumptions, the second representation for $\overline{p F}(t)$ in Eq. (2.1) becomes

$$
\begin{aligned}
\overline{p F}(t) & =\sum_{k=1}^{n S L}\left(\int_{0}^{t}[p(\tau)]^{n S L-1}[1-p(\tau)]^{n W L} p^{\prime}(\tau) \mathrm{d} \tau\right) \\
& =(n S L) \int_{0}^{t}[p(\tau)]^{n S L-1}[1-p(\tau)]^{n W L} p^{\prime}(\tau) \mathrm{d} \tau \\
& =(n S L) \int_{p(0)}^{p(t)} p^{n S L-1}(1-p)^{n W L} \mathrm{~d} p .
\end{aligned}
$$

In turn, 


$$
\begin{aligned}
\overline{p F}(\infty) & =\lim _{t \rightarrow \infty}(n S L) \int_{p(0)}^{p(t)} p^{n S L-1}(1-p)^{n W L} \mathrm{~d} p \\
& =(n S L) \int_{0}^{1} p^{n S L-1}(1-p)^{n W L} \mathrm{~d} p \\
& =(n S L)[(n S L-1) ! n W L ! /(n S L+n W L) !] \\
& =n S L ! n W L !(n S L+n W L) ! .
\end{aligned}
$$

The crucial third equality in the preceding expression follows from the identity

$$
\int_{0}^{1} x^{m}(1-x)^{n} d x=\Gamma(m+1) \Gamma(n+1) / \Gamma(m+n+2)
$$

for $m>-1, n>-1$ and $\Gamma$ denoting the gamma function (Eq. (586), p. 396, Ref. [26]). As a reminder, $\Gamma(m+1)=m$ ! if $m \geq 0$ is an integer (Sect. 6.11.3, Ref. [26]).

The relationship in Eq. (2.5) results in very simple and easily determined representations for PLOAS with different numbers of WLs and SLs when an analysis is carried out to the time at which all links have failed (Table 1). Specifically, PLOAS can be calculated for a variety of values for $n W L$ and $n S L$ and the results compared with the values in Table 1. Failure to agree with the PLOAS values in Table 1 indicates that there must be an error somewhere in the analysis.

As an example, the results in Table 1 can be used to verify representations for PLOAS obtained in Ref. [27] and explicitly stated in Table 2. As examination of Table 2 shows, the representations for PLOAS are fairly complex and certainly too complex for closed form evaluation. Two quadrature-based procedures (i.e., trapezoidal method and Simpson's method) and two sampling-based procedures (i.e., simple random sampling and importance sampling) are developed in Ref. [27] for the evaluation of the integrals in Table 2 that define PLOAS. In turn, these procedures are implemented in the CPLOAS program (App. III, Ref. [11]).

For this verification, the representations for PLOAS in Table 2 must be formulated in a manner that is equivalent to the representations for PLOAS in Eq. (2.1). This can be accomplished by assigning the same temperature curve and the same failure temperature density function to all links. Then, all links have the same cumulative distribution for failure time, which is the basis for the representations of PLOAS in Eq. (2.5) and hence the results in Table 1.

To have a specific example, the time-temperature curve for the WL in Fig. 4 of Ref. [27] and the associated density function for failure temperature are used for illustration. Specifically, this results in temperature curves defined by 
Table 1. Values for PLOAS (i.e., $\overline{p F}(\infty)$ in Eq. (2.5)) for Different Numbers of WLs and SLs Predicated on the Assumptions that (i) Loss of Assured Safety Corresponds to Failure of All SLs Before Failure of Any WL, (ii) The Failures of the Individual Links are Independent, and (iii) All Links Have the Same Distribution for Failure Time

\begin{tabular}{|c|c|c|c|c|c|}
\hline \multicolumn{7}{|c|}{ Integer Ratio Representation } \\
\hline $\boldsymbol{n} \boldsymbol{S} \boldsymbol{L} \boldsymbol{n} \boldsymbol{W L}$ & $\mathbf{1}$ & $\mathbf{2}$ & $\mathbf{3}$ & $\mathbf{4}$ & $\mathbf{5}$ \\
\hline $\mathbf{1}$ & $1 / 2$ & $1 / 3$ & $1 / 4$ & $1 / 5$ & $1 / 6$ \\
\hline $\mathbf{2}$ & $1 / 3$ & $1 / 6$ & $1 / 10$ & $1 / 15$ & $1 / 21$ \\
\hline $\mathbf{3}$ & $1 / 4$ & $1 / 10$ & $1 / 20$ & $1 / 35$ & $1 / 56$ \\
\hline $\mathbf{4}$ & $1 / 5$ & $1 / 15$ & $1 / 35$ & $1 / 70$ & $1 / 126$ \\
\hline $\mathbf{5}$ & $1 / 6$ & $1 / 21$ & $1 / 56$ & $1 / 126$ & $1 / 252$ \\
\hline \multicolumn{7}{|c|}{ Decimal Fraction Representation } \\
\hline $\boldsymbol{n} \boldsymbol{S} \mathbf{n} \boldsymbol{W L}$ & $\mathbf{1}$ & $\mathbf{2}$ & $\mathbf{3}$ & $\mathbf{4}$ & $\mathbf{5}$ \\
\hline $\mathbf{1}$ & 0.50000 & 0.33333 & 0.25000 & 0.20000 & 0.16667 \\
\hline $\mathbf{2}$ & 0.33333 & 0.16667 & 0.10000 & 0.06667 & 0.04762 \\
\hline $\mathbf{3}$ & 0.25000 & 0.10000 & 0.05000 & 0.02857 & 0.01786 \\
\hline $\mathbf{4}$ & 0.20000 & 0.06667 & 0.02857 & 0.01429 & 0.00794 \\
\hline $\mathbf{5}$ & 0.16667 & 0.04762 & 0.01786 & 0.00794 & 0.00397 \\
\hline
\end{tabular}

$$
T M P W L_{j}(t)=T M P S L_{k}(t)=c_{1}+\left[c_{2}+c_{3} \exp \left(-c_{4} t\right) \sin \left(c_{5} t\right)\right] \tanh \left(c_{6} t\right)
$$

for $0 \leq t \leq 100 \mathrm{~min}, c_{1}=10^{\circ} \mathrm{C}, c_{2}=900^{\circ} \mathrm{C}, c_{3}=-1000{ }^{\circ} \mathrm{C}, c_{4}=0.30 \mathrm{~min}^{-1}, c_{5}=0.17 \mathrm{~min}^{-1}$, and $c_{6}=0.03 \mathrm{~min}^{-1}$ (see Eq. (2.66), Ref. [27]), and failure temperature density functions defined by

$$
f W L_{j}(T)=f S L_{k}(T)=\left(1 / c_{9} \sqrt{2 \pi}\right) \exp \left[-\left(T-c_{8}\right)^{2} / 2 c_{9}^{2}\right]
$$

for $c_{8}=310^{\circ} \mathrm{C}$ and $c_{9}=8^{\circ} \mathrm{C}$ (see Eq. (2.68), Ref. [11]).

The CPLOAS program was then used to determine PLOAS with the indicated temperature curves and density functions for all combinations of WLs and SLs indicated in Table 1. Further, calculations were carried out with each of the previously indicated integration procedures (i.e., trapezoidal method, Simpson's method, simple random sampling, and importance sampling). The outcome was that the two quadrature methods produced results for all WL/SL combinations that agreed identically with the decimal representations for PLOAS in Table 1 . The two sampling-based methods produced results that were close to the decimal values in Table 1 but, as would be expected, were not in complete agreement (Table 3 ). 
Table 2. Representation of Value $p F$ for PLOAS Under Fire Conditions for WL/SL System With $n W L$ WLs, nSL SLs, and the Assumptions that (i) a Link Fails Instantly When it Reaches its Failure Temperature and (ii) Loss of Assured Safety Corresponds to Failure of all SLs Before Failure of Any WL

$$
\begin{aligned}
& p F=\sum_{k=1}^{n S L}\left(\int_{t M I N}^{t M A X}\left\{f S L_{k}\left[T M P S L_{k}(t)\right]\right\}\left\{\prod_{\substack{l=1 \\
l \neq k}}^{n S L} I\left[-\infty, T M P S L_{l}(t), f S L_{l}\right]\right\}\right. \\
& \left.\times\left\{\prod_{j=1}^{n W L} I\left[T M P W L_{j}(t), \infty, f W L_{j}\right]\right\} \mathrm{d} T M P S L_{k}(t)\right) \\
& =\int_{t M I N}^{t M A X}\left(\sum_{k=1}^{n S L}\left\{f S L_{k}\left[T M P S L_{k}(t)\right]\right\}\left\{\prod_{\substack{l=1 \\
l \neq k}}^{n S L} I\left[-\infty, T M P S L_{l}(t), f S L_{l}\right]\right\}\left\{\mathrm{d} T M P S L_{k}(t) / \mathrm{d} t\right\}\right) \\
& \times\left(\prod_{j=1}^{n W L} I\left[T M P W L_{j}(t), \infty, f W L_{j}\right]\right) \mathrm{d} t \\
& =\sum_{k=1}^{n S L} \int_{T M N S L_{k}}^{T M X S L_{k}}\left\{\left\{f S L_{k}\left(T_{S L}\right)\right\}\left\{\prod_{\substack{l=1 \\
l \neq k}}^{n S L} I\left[-\infty, T M P S L_{l}\left[T M P S L_{k}^{-1}\left(T_{S L}\right)\right], f S L_{l}\right]\right\}\right. \\
& \left.\times\left\{\prod_{j=1}^{n W L} I\left[T M P W L_{j}\left[T M P S L_{k}^{-1}\left(T_{S L}\right)\right], \infty, f W L_{j}\right]\right\}\right) \mathrm{d} T_{S L}
\end{aligned}
$$

where

$$
\begin{aligned}
I[a, b, f] & =\int_{a}^{b} f(T) d T \\
f W L_{j}\left(T_{W L}\right) & =\text { density function }\left({ }^{\circ} \mathrm{C}^{-1}\right) \text { for failure temperature of } \mathrm{WL} j, \\
f S L_{k}\left(T_{S L}\right) & =\text { density function }\left({ }^{\circ} \mathrm{C}^{-1}\right) \text { for failure temperature of SL } k, \\
T M P W L_{j}(t) & =\text { temperature }\left({ }^{\circ} \mathrm{C}\right) \text { of } \mathrm{WL} j \text { at time } t \text { for } t M I N \leq t \leq t M A X, \\
T M P S L_{k}(t) & =\text { temperature }\left({ }^{\circ} \mathrm{C}\right) \text { of } \mathrm{SL} k \text { at time } t \text { for } t M I N \leq t \leq t M A X, \\
T M N S L_{k} & =T M P S L_{k}(t M I N), \\
T M X S L_{k} & =T M P S L_{k}(t M A X)
\end{aligned}
$$

For perspective, the importance sampling calculations were implemented with two different importance sampling distributions for failure temperature: (i) uniform on $\left[c_{8}-4 c_{9}, c_{8}+4 c_{9}\right]=\left[272,342{ }^{\circ} \mathrm{C}\right]$, which emphasizes the tails of the failure temperature distribution, and (ii) normal with $\mu=c_{8}=310{ }^{\circ} \mathrm{C}$ and $\sigma=c_{9} / 2=4{ }^{\circ} \mathrm{C}$, which emphasizes the central part of the failure temperature distribution. In this example, emphasizing the central part of 
the failure temperature distribution produced more accurate results than emphasizing the tails of the failure temperature distribution (Table 3). However, no attempt was made to optimize the importance sampling strategy in use. As a reminder, importance sampling has the potential to either enhance or retard convergence relative to results obtained with simple random sampling depending on the specific sampling strategy selected for use. Additional information on importance sampling is available in a number of references. ${ }^{28-36}$

As a reminder, the PLOAS calculations being carried out by the CPLOAS program involve the full numerical evaluation of the defining integrals in Table 2. Thus, even though the PLOAS values under consideration have simple algebraic representations, full numerical integrations are being carried out in the CPLOAS program. As a result, agreement between the results calculated with CPLOAS and the analytically derived results in Table 1 helps provide assurance that both the derived representations for PLOAS in Table 2 and the numerical approximations to these representations are correct.

This section concludes by comparing the representation for PLOAS in Eq. (4.1) with an earlier representation developed by Mike Bohn (App. I, Ref. [11]) that motivated the development of the verification test problems in this presentation. The Bohn development considers one WL and $n S L$ SLs, with the corresponding PLOAS representation $\overline{p F}(t)$ for one WL and two SLs shown in Eq. (7.1) of Ref. [11]. For comparison, the second representation for $\overline{p F}(t)$ in Eq. (2.1) with one WL and two SLs reduces to

$$
\overline{p F}(t)=\sum_{k=1}^{2}\left(\int_{0}^{t}\left\{\prod_{\substack{l=1 \\ l \neq k}}^{2} C D F_{S L, l}(\tau)\right\}\left\{1-C D F_{W L, 1}(\tau)\right\}\left\{\mathrm{d} C D F_{S L, k}(\tau) / \mathrm{d} \tau\right\} \mathrm{d} \tau\right)
$$

which is not quite the same as the Bohn representation in Eq. (7.1) of Ref. [11] for the corresponding WL/SL configuration.

The reason for this difference is that the representation for $\overline{p F}(t)$ in Eq. (7.1) of Ref. [11] is only an approximation to the formally correct value for $\overline{p F}(t)$ in Eq. (2.9). In particular, the representation for $\overline{p F}(t)$ in Eq. (7.1) of Ref. [11] does not fully incorporate the probability associated with WL failure after the failure of all SLs. More specifically, the Riemann-Stieltjes integral representation for $\overline{p F}(t)$ in Eq. (7.1) of Ref. [11] does not incorporate the probability associated with failure patterns in which all SLs fail before time $t$ and the WL fails at a time significantly later than time $t$. The result of this lost probability is that $\overline{p F}(t)$ as defined in Eq. (7.1) of Ref. [11] is actually an underestimate of PLOAS at early times. However, as time increases, this underestimate decreases and the value for $\overline{p F}(t)$ in Eq. (7.1) of Ref. [11] converges to the value in Eq. (2.9). The representation for $\overline{p F}(t)$ in Eqs. (2.1) and (2.9) avoids the indicated underestimation for PLOAS through the inclusion of the factor $\{\sim\}_{2}$ in Eq. (2.2). 
Table 3. Deviations from Decimal Results in Table 1 Observed in Determination of PLOAS with Sampling-Based Procedures and Samples of Size $10^{6}$

\begin{tabular}{|c|c|c|c|c|c|}
\hline \multicolumn{6}{|c|}{ Random Sampling } \\
\hline$n S L / n W L$ & 1 & 2 & 3 & 4 & 5 \\
\hline 1 & 0.00038 & -0.00005 & -0.00052 & -0.00004 & -0.00018 \\
\hline 2 & 0.00005 & -0.00037 & 0.00037 & 0.00021 & -0.00002 \\
\hline 3 & -0.00043 & 0.00013 & -0.00028 & -0.00024 & -0.00003 \\
\hline 4 & 0.00018 & 0.00005 & -0.00027 & -0.00002 & 0.00002 \\
\hline 5 & 0.00002 & 0.00000 & 0.00003 & -0.00007 & 0.00008 \\
\hline \multicolumn{6}{|c|}{ Importance Sampling: Uniform on $\left[c_{8}-4 c_{9}, c_{8}+4 c_{9}\right]=\left[271,342^{\circ} \mathrm{C}\right]$} \\
\hline$n S L / n W L$ & 1 & 2 & 3 & 4 & 5 \\
\hline 1 & 0.00064 & -0.00145 & -0.00329 & -0.00031 & -0.00804 \\
\hline 2 & 0.00043 & -0.00149 & -0.00028 & -0.00050 & 0.00276 \\
\hline 3 & -0.00114 & -0.00028 & -0.00182 & -0.00379 & 0.00693 \\
\hline 4 & -0.00252 & -0.00256 & 0.00319 & 0.00226 & -0.00166 \\
\hline 5 & -0.00373 & 0.00157 & 0.00485 & -0.00417 & 0.00320 \\
\hline \multicolumn{6}{|c|}{ Importance Sampling: Normal with $\mu=c_{8}=310^{\circ}, \sigma=c_{9} / 2=4^{\circ} \mathrm{C}$} \\
\hline$n S L / n W L$ & 1 & 2 & 3 & 4 & 5 \\
\hline 1 & 0.00044 & -0.00010 & -0.00053 & 0.00025 & -0.00027 \\
\hline 2 & -0.00019 & -0.00025 & 0.00035 & 0.00032 & -0.00007 \\
\hline 3 & -0.00050 & 0.00021 & -0.00018 & -0.00038 & 0.00002 \\
\hline 4 & 0.00037 & 0.00017 & -0.00033 & 0.00002 & 0.00002 \\
\hline 5 & 0.00007 & 0.00005 & 0.00009 & -0.00006 & 0.00011 \\
\hline
\end{tabular}


This page intentionally left blank. 


\section{Problem 2: Failure of Any SL Before Failure of Any WL}

Verification Test Problem 2 is predicated on the assumption that loss of assured safety corresponds to failure of any SL before failure of any WL and the additional assumption that the failures of the individual links are independent. With the indicated assumptions,

$$
\begin{aligned}
\overline{p F}(t) & =\sum_{k=1}^{n S L}\left(\int_{0}^{t}\left\{\prod_{\substack{l=1 \\
l \neq k}}^{n S L}\left[1-C D F_{S L, l}(\tau)\right]\right\}\left\{\prod_{j=1}^{n W L}\left[1-C D F_{W L, j}(\tau)\right]\right\} \mathrm{d} C D F_{S L, k}(\tau)\right) \\
& =\sum_{k=1}^{n S L}\left(\int_{0}^{t}\left\{\prod_{\substack{l=1 \\
l \neq k}}^{n S L}\left[1-C D F_{S L, l}(\tau)\right]\right\}\left\{\prod_{j=1}^{n W L}\left[1-C D F_{W L, j}(\tau)\right]\right\}\left\{\mathrm{d} C D F_{S L, k}(\tau) / \mathrm{d} \tau\right\} \mathrm{d} \tau\right),
\end{aligned}
$$

where the first integral is a Riemann-Stieltjes integral, the second integral is the corresponding Riemann integral, and $C D F_{W L, j}(\tau)$ and $C D F_{S L, k}(\tau)$ are defined the same as in Eq. (2.1).

The integrals defining $\overline{p F}(t)$ in Eq. (3.1) are obtained from the approximations

$$
\begin{aligned}
\overline{p F}(t) & \cong \sum_{k=1}^{n S L}\left(\sum_{i=1}^{n}\left\{\prod_{\substack{l=1 \\
l \neq k}}^{n S L}\left[1-C D F_{S L, l}\left(\tau_{i}\right)\right]\right\}\left\{\prod_{j=1}^{n W L}\left[1-C D F_{W L, j}\left(\tau_{i}\right)\right]\right\}_{2}\left\{C D F_{S L, k}\left(\tau_{i}\right)-C D F_{S L, k}\left(\tau_{i-1}\right)\right\}_{3}\right) \\
& =\sum_{k=1}^{n S L}\left(\sum_{i=1}^{n}\left\{\prod_{\substack{l=1 \\
l \neq k}}^{n S L}\left[1-C D F_{S L, l}\left(\tau_{i}\right)\right]\right\}\left\{\prod_{j}^{n W L}\left[1-C D F_{W L, j}\left(\tau_{i}\right)\right]\right\}_{2}\left\{\frac{C D F_{S L, k}\left(\tau_{i}\right)-C D F_{S L, k}\left(\tau_{i-1}\right)}{\Delta \tau_{i}}\right\}_{4} \Delta \tau_{i}\right)
\end{aligned}
$$

for $0=\tau_{0}<\tau_{1}<\ldots<\tau_{n}=t$ and $\Delta \tau_{i}=\tau_{i}-\tau_{i-1}$. Specifically, $\{\sim\}_{1}$ is the probability that all SLs except SL $k$ fail after time $\tau_{i} ;\{\sim\}_{2}$ is the probability that all WLs fail after time $\tau_{i} ;\{\sim\}_{3}$ is the probability that SL $k$ fails between $\tau_{i-1}$ and $\tau_{i}$; and $\{\sim\}_{4}$ is an approximation to $\mathrm{d} C D F_{S L, k}(\tau) / \mathrm{d}(\tau)$. The integrals in Eq. (3.1) then result in the limit as $\Delta \tau_{i}$ goes to zero.

As in Eqs. (2.4) and (2.5), the desired verification test results are obtained by assuming all links have the same distribution for failure time. Specifically, the cumulative distribution of failure time for each link is assumed to be defined by the function $p(\tau)$ indicated in Eq. (2.3). With the preceding assumption, 


$$
\begin{aligned}
\overline{p F}(t) & =\sum_{k=1}^{n S L}\left(\int_{0}^{t}[1-p(t)]^{n S L-1}[1-p(t)]^{n W L} p^{\prime}(\tau) \mathrm{d} \tau\right) \\
& =(n S L) \int_{0}^{t}[1-p(t)]^{n W L+n S L-1} p^{\prime}(\tau) \mathrm{d} \tau \\
& =(n S L) \int_{p(0)}^{p(t)}[1-p]^{n W L+n S L-1} \mathrm{~d} p .
\end{aligned}
$$

In turn,

$$
\begin{aligned}
\overline{p F}(\infty) & =\lim _{t \rightarrow \infty}(n S L) \int_{p(0)}^{p(t)}[1-p]^{n W L+n S L-1} \mathrm{~d} p \\
& =(n S L) \int_{0}^{1} p^{0}[1-p]^{n W L+n S L-1} \mathrm{~d} p \\
& =(n S L)[0 !(n W L+n S L-1) ! /(0+n W L+n S L-1+1) !] \\
& =n S L /(n W L+n S L),
\end{aligned}
$$

where the third equality follows from Eq. (2.6).

The relationship in Eq. (3.4) results in very simple and easily determined representations for PLOAS with different numbers of WLs and SLs when an analysis is carried out to the time at which all links have failed (Table 4). As an example, the results in Table 4 can be used to verify representations for PLOAS obtained in Ref. [27] and explicitly stated in Table 5. For this verification, all links are assumed to have the temperature curve and failure temperature density function defined in Eqs. (2.7) and (2.8).

The CPLOAS program was used to determine PLOAS with the indicated temperature curves and density functions for all combinations of WLs and SLs indicated in Table 4. Further, calculations were carried out with each of the integration procedures implemented in CPLOAS (i.e., trapezoidal method, Simpson's method, simple random sampling, and importance sampling). The outcome was that the two quadrature methods produced results for all WL/SL combinations that agreed identically with the decimal representations for PLOAS in Table 4. The two sampling-based methods produced results that were close to the decimal values in Table 4 but, as would be expected, were not in complete agreement (Table 6). The observed agreement with the verification test problem provides a strong indication that the derivation of the results in Table 5 and the numerical evaluation of the associated integrals in CPLOAS are correct. 
Table 4. Values for PLOAS (i.e., $\overline{p F}(\infty)$ in Eq. (3.4)) for Different Numbers of WLs and SLs Predicated on the Assumptions that (i) Loss of Assured Safety Corresponds to Failure of Any SL Before Failure of Any WL, (ii) the Failures of the Individual Links are Independent, and (iii) All Links Have the Same Distribution for Failure Time.

\begin{tabular}{|c|c|c|c|c|c|}
\hline \multicolumn{7}{|c|}{ Integer Ratio Representation } \\
\hline $\boldsymbol{n S} \boldsymbol{L} \boldsymbol{n} \boldsymbol{W L}$ & $\mathbf{1}$ & $\mathbf{2}$ & $\mathbf{3}$ & $\mathbf{4}$ & $\mathbf{5}$ \\
\hline $\mathbf{1}$ & $1 / 2$ & $1 / 3$ & $1 / 4$ & $1 / 5$ & $1 / 6$ \\
\hline $\mathbf{2}$ & $2 / 3$ & $2 / 4$ & $2 / 5$ & $2 / 6$ & $2 / 7$ \\
\hline $\mathbf{3}$ & $3 / 4$ & $3 / 5$ & $3 / 6$ & $3 / 7$ & $3 / 8$ \\
\hline $\mathbf{4}$ & $4 / 5$ & $4 / 6$ & $4 / 7$ & $4 / 8$ & $4 / 9$ \\
\hline $\mathbf{5}$ & $5 / 6$ & $5 / 7$ & $5 / 8$ & $5 / 9$ & $5 / 10$ \\
\hline \multicolumn{7}{|c|}{ Decimal Fraction Representation } \\
\hline $\boldsymbol{n} \boldsymbol{L} / \boldsymbol{n} \boldsymbol{W L}$ & $\mathbf{1}$ & $\mathbf{2}$ & $\mathbf{3}$ & $\mathbf{4}$ & $\mathbf{5}$ \\
\hline $\mathbf{1}$ & 0.50000 & 0.33333 & 0.25000 & 0.20000 & 0.16667 \\
\hline $\mathbf{2}$ & 0.66667 & 0.50000 & 0.40000 & 0.33333 & 0.28571 \\
\hline $\mathbf{3}$ & 0.75000 & 0.60000 & 0.50000 & 0.42857 & 0.37500 \\
\hline $\mathbf{4}$ & 0.80000 & 0.66667 & 0.57143 & 0.50000 & 0.44444 \\
\hline $\mathbf{5}$ & 0.83333 & 0.71429 & 0.62500 & 0.55556 & 0.50000 \\
\hline
\end{tabular}


Table 5. Representation of Value $p F$ for PLOAS Under Fire Conditions for WL/SL System With $n W L$ WLs, $n S L$ SLs, and the Assumptions that (i) a Link Fails Instantly When it Reaches its Failure Temperature and (ii) Loss of Assured Safety Corresponds to Failure of Any SL Before Failure of Any WL

$$
\begin{aligned}
& p F=\sum_{k=1}^{n S L}\left(\int_{t M I N}^{t M A X}\left\{f S L_{k}\left[T M P S L_{k}(t)\right]\right\}\left\{\prod_{\substack{l=1 \\
l \neq k}}^{n S L} I\left[T M P S L_{l}(t), \infty, f S L_{l}\right]\right\}\right. \\
& \left.\times\left\{\prod_{j=1}^{n W L} I\left[T M P W L_{j}(t), \infty, f W L_{j}\right]\right\} \mathrm{d} T M P S L_{k}(t)\right) \\
& =\int_{t M I N}^{t M A X}\left(\sum_{k=1}^{n S L}\left\{f S L_{k}\left[T M P S L_{k}(t)\right]\right\}\left\{\prod_{\substack{l=1 \\
l \neq k}}^{n S L} I\left[T M P S L_{l}(t), \infty, f S L_{l}\right]\right\}\left\{\mathrm{d} T M P S L_{k}(t) / \mathrm{d} t\right\}\right) \\
& \times\left(\prod_{j=1}^{n W L} I\left[T M P W L_{j}(t), \infty, f W L_{j}\right]\right) \mathrm{d} t \\
& =\sum_{k=1}^{n S L} \int_{T M N S L_{k}}^{T M X S L_{k}}\left\{\left\{f S L_{k}\left(T_{S L}\right)\right\}\left\{\prod_{\substack{l=1 \\
l \neq k}}^{n S L} I\left[T M P S L_{l}\left[\operatorname{TMPSL}_{k}^{-1}\left(T_{S L}\right)\right], \infty, f S L_{l}\right]\right\}\right. \\
& \times\left\{\prod_{j=1}^{n W L} I\left[T M P W L_{j}\left[\operatorname{TMPSL}_{k}^{-1}\left(T_{S L}\right)\right], \infty, f W L_{j}\right]\right\} \mathrm{d} T_{S L}
\end{aligned}
$$

with same notation as in Table 2. 
Table 6. Deviations from Decimal Results in Table 4 Observed in Determination of PLOAS with Sampling-Based Procedures and Samples of Size $10^{6}$

\begin{tabular}{|c|c|c|c|c|c|}
\hline \multicolumn{6}{|c|}{ Random Sampling } \\
\hline$n S L / n W L$ & 1 & 2 & 3 & 4 & 5 \\
\hline 1 & 0.00038 & -0.00005 & -0.00052 & -0.00004 & -0.00018 \\
\hline 2 & 0.00077 & -0.00089 & -0.00013 & 0.00011 & 0.00023 \\
\hline 3 & -0.00007 & 0.00002 & 0.00075 & 0.00011 & -0.00029 \\
\hline 4 & -0.00039 & -0.00016 & 0.00012 & -0.00028 & -0.00042 \\
\hline 5 & 0.00012 & -0.00036 & -0.00053 & -0.00080 & 0.00025 \\
\hline \multicolumn{6}{|c|}{ Importance Sampling: Uniform on $\left[c_{8}-4 c_{9}, c_{8}+4 c_{9}\right]=\left[271,342^{\circ} \mathrm{C}\right]$} \\
\hline$n S L / n W L$ & 1 & 2 & 3 & 4 & 5 \\
\hline 1 & 0.00064 & -0.00145 & -0.00329 & -0.00031 & -0.00804 \\
\hline 2 & 0.00128 & -0.00174 & 0.00171 & -0.01008 & 0.00265 \\
\hline 3 & 0.00026 & 0.00289 & -0.01024 & -0.00067 & 0.00593 \\
\hline 4 & 0.00041 & -0.01445 & -0.00032 & -0.00125 & -0.01419 \\
\hline 5 & -0.00962 & 0.00573 & -0.00141 & -0.00917 & 0.00059 \\
\hline \multicolumn{6}{|c|}{ Importance Sampling: Normal with $\mu=c_{8}=310^{\circ}, \sigma=c_{9} / 2=4^{\circ} \mathrm{C}$} \\
\hline$n S L / n W L$ & 1 & 2 & 3 & 4 & 5 \\
\hline 1 & 0.00044 & -0.00010 & -0.00053 & 0.00025 & -0.00027 \\
\hline 2 & 0.00085 & -0.00103 & 0.00004 & 0.00001 & 0.00008 \\
\hline 3 & -0.00019 & -0.00004 & 0.00053 & 0.00007 & -0.00033 \\
\hline 4 & -0.00042 & -0.00048 & 0.00010 & -0.00050 & -0.00034 \\
\hline 5 & -0.00010 & -0.00028 & -0.00080 & -0.00060 & 0.00015 \\
\hline
\end{tabular}


This page intentionally left blank. 


\section{Problem 3: Failure of All SLs Before Failure of All WLs}

Verification Test Problem 3 is predicated on the assumptions that loss of assured safety corresponds to failure of all SLs before failure of all WLs and the additional assumption that the failures of the individual links are independent. With the indicated assumptions,

$$
\begin{aligned}
p F(t) & =\sum_{k=1}^{n S L}\left(\int_{0}^{t}\left\{\prod_{\substack{l=1 \\
l \neq k}}^{n S L} C D F_{S L, l}(\tau)\right\}\left\{1-\prod_{j=1}^{n W L} C D F_{W L, j}(\tau)\right\} \mathrm{d} C D F_{S L, k}(\tau)\right) \\
& =\sum_{k=1}^{n S L}\left(\int_{0}^{t}\left\{\prod_{\substack{l=1 \\
l \neq k}}^{n S L} C D F_{S L, l}(\tau)\right\}\left\{1-\prod_{j=1}^{n W L} C D F_{W L, j}(\tau)\right\}\left\{\mathrm{d} C D F_{S L, k}(\tau) / \mathrm{d} \tau\right\} \mathrm{d} \tau\right),
\end{aligned}
$$

where the first integral is a Riemann-Stieltjes integral, the second integral is the corresponding Riemann integral, and $C D F_{W L, j}(\tau)$ and $C D F_{S L, k}(\tau)$ are defined the same as in Eq. (2.1).

The integrals defining $\overline{p F}(t)$ in Eq. (4.1) are obtained from the approximations

$$
\begin{aligned}
& \overline{p F}(t) \cong \sum_{k=1}^{n S L}\left(\sum_{i=1}^{n}\left\{\prod_{\substack{l=1 \\
l \neq k}}^{n S L} C D F_{S L, l}\left(\tau_{i-1}\right)\right\}_{1}\left\{1-\prod_{j=1}^{n W L} C D F_{W L, j}\left(\tau_{i-1}\right)\right\}_{2}\left\{C D F_{S L, k}\left(\tau_{i}\right)-C D F_{S L, k}\left(\tau_{i-1}\right)\right\}_{3}\right) \\
& =\sum_{k=1}^{n S L}\left(\sum_{i=1}^{n}\left\{\prod_{\substack{l=1 \\
l \neq k}}^{n S L} C D F_{S L, l}\left(\tau_{i-1}\right)\right\}_{1}\left\{1-\prod_{j=1}^{n W L} C D F_{W L, j}\left(\tau_{i-1}\right)\right\}_{2}\left\{\frac{C D F_{S L, k}\left(\tau_{i}\right)-C D F_{S L, k}\left(\tau_{i-1}\right)}{\Delta \tau_{i}}\right\}_{4} \Delta \tau_{i}\right)
\end{aligned}
$$

for $0=\tau_{0}<\tau_{1}<\ldots<\tau_{n}=t$ and $\Delta \tau_{i}=\tau_{i}-\tau_{i-1}$. Specifically, $\{\sim\}_{1}$ is the probability that all SLs except SL $k$ fail before time $\tau_{i-1} ;\{\sim\}_{2}$ is the probability that at least one WL has not failed by time $\tau_{i-1} ;\{\sim\}_{3}$ is the probability that SL $k$ fails between $\tau_{i-1}$ and $\tau_{i}$; and $\{\sim\}_{4}$ is an approximation to $\mathrm{d} C D F_{S L, k}(\tau) / \mathrm{d} \tau$. The integrals in Eq. (4-1) then result in the limit as $\Delta \tau_{i}$ goes to zero.

Similarly to the results in Eqs. (2.4), (2.5), (3.3) and (3.4) and with $p(\tau)$ defined the same as in Eq. (2.3), 


$$
\begin{aligned}
\overline{p F}(t) & =\sum_{k=1}^{n S L}\left(\int_{0}^{t}\left\{[p(\tau)]^{n S L-1}\right\}\left\{1-[p(\tau)]^{n W L}\right\} p^{\prime}(\tau) \mathrm{d} \tau\right) \\
& =(n S L) \int_{0}^{t}\left\{[p(\tau)]^{n S L-1}-[p(\tau)]^{n W L+n S L-1}\right\} p^{\prime}(\tau) \mathrm{d} \tau \\
& =(n S L) \int_{p(0)}^{p(t)}\left(p^{n S L-1}-p^{n W L+n S L-1}\right) \mathrm{d} p .
\end{aligned}
$$

In turn,

$$
\begin{aligned}
\overline{p F}(\infty) & =\lim _{t \rightarrow \infty}(n S L) \int_{p(0)}^{p(t)}\left(p^{n S L-1}-p^{n W L+n S L-1}\right) \mathrm{d} p \\
& =(n S L) \int_{0}^{1}\left(p^{n S L-1}-p^{n W L+n S L-1}\right) \mathrm{d} p \\
& =(n S L)\left(\int_{0}^{1} p^{n S L-1}(1-p)^{0} \mathrm{~d} p-\int_{0}^{1} p^{n W L+n S L-1}(1-p)^{0} \mathrm{~d} p\right) \\
& =(n S L)([(n S L-1) ! 0 ! /(n S L+0) !]-[(n W L+n S L-1) ! 0 ! /(n W L+n S L+0) !]) \\
& =(n S L)([1 / n S L]-[1 /(n W L+n S L)]) \\
& =n W L /(n W L+n S L),
\end{aligned}
$$

where the fourth equality follows from Eq. (2.6).

The relationship in Eq. (4.4) results in very simple and easily determined representations for PLOAS with different numbers of WLs and SLs when an analysis is carried out to the time at which all links have failed (Table 7). As an example, the results in Table 7 can be used to verify the representations for PLOAS shown in Table 8. The results in Table 8 are not included in the PLOAS representations given in Ref. [27] but can be derived in a similar manner. For this verification, all links are assumed to have the temperature curve and failure temperature density function defined in Eqs. (2.7) and (2.8).

The CPLOAS program was used to determine PLOAS with the indicated temperature curves and density functions for all combinations of WLs and SLs in Table 7. Further, calculations were carried out with each of the integration procedures implemented in CPLOAS (i.e., trapezoidal method, Simpson's method, simple random sampling, and importance sampling). The outcome was that the two quadrature methods produced results for all WL/SL combinations that agreed identically with the decimal representations for PLOAS in Table 7. The two sampling-based methods produced results that were close to the decimal values in Table 7 but, as would be expected, were not in complete agreement (Table 9). 
Table 7. Values for PLOAS (i.e., $\overline{p F}(\infty)$ in Eq. (4.4)) for Different Numbers of WLs and SLs Predicated on the Assumptions that (i) Loss of Assured Safety Corresponds to Failure of All SLs Before Failure of All WLs, (ii) the Failures of the Individual Links are Independent, and (iii) All Links Have the Distribution for Failure Time

\begin{tabular}{|c|c|c|c|c|c|}
\hline \multicolumn{7}{|c|}{ Integer Ratio Representation } \\
\hline $\boldsymbol{n} \boldsymbol{S} \boldsymbol{h} \boldsymbol{n} \boldsymbol{W} \boldsymbol{L}$ & $\mathbf{1}$ & $\mathbf{2}$ & $\mathbf{3}$ & $\mathbf{4}$ & $\mathbf{5}$ \\
\hline $\mathbf{1}$ & $1 / 2$ & $2 / 3$ & $3 / 4$ & $4 / 5$ & $5 / 6$ \\
\hline $\mathbf{2}$ & $1 / 3$ & $2 / 4$ & $3 / 5$ & $4 / 6$ & $5 / 7$ \\
\hline $\mathbf{3}$ & $1 / 4$ & $2 / 5$ & $3 / 6$ & $4 / 7$ & $5 / 8$ \\
\hline $\mathbf{4}$ & $1 / 5$ & $2 / 6$ & $3 / 7$ & $4 / 8$ & $5 / 9$ \\
\hline $\mathbf{5}$ & $1 / 6$ & $2 / 7$ & $3 / 8$ & $4 / 9$ & $5 / 10$ \\
\hline \multicolumn{7}{|c|}{ Decimal Fraction Representation } \\
\hline $\boldsymbol{n} \boldsymbol{S} / \boldsymbol{n} \boldsymbol{W L}$ & $\mathbf{1}$ & $\mathbf{2}$ & $\mathbf{3}$ & $\mathbf{4}$ & $\mathbf{5}$ \\
\hline $\mathbf{1}$ & 0.50000 & 0.66667 & 0.75000 & 0.80000 & 0.83333 \\
\hline $\mathbf{2}$ & 0.33333 & 0.50000 & 0.60000 & 0.66667 & 0.71429 \\
\hline $\mathbf{3}$ & 0.25000 & 0.40000 & 0.50000 & 0.57143 & 0.62500 \\
\hline $\mathbf{4}$ & 0.20000 & 0.33333 & 0.42857 & 0.50000 & 0.55556 \\
\hline $\mathbf{5}$ & 0.16667 & 0.28571 & 0.37500 & 0.44444 & 0.50000 \\
\hline
\end{tabular}


Table 8. Representation of Value $p F$ for PLOAS Under Fire Conditions for WL/SL System With $n W L$ WLs, $n S L$ SLs, and the Assumptions that (i) a Link Fails Instantly When it Reaches its Failure Temperature and (ii) Loss of Assured Safety Corresponds to Failure of All SLs Before Failure of All WL

$$
\begin{aligned}
& p F=\sum_{k=1}^{n S L}\left(\int_{t M I N}^{t M A X}\left\{f S L_{k}\left[T M P S L_{k}(t)\right]\right\}\left\{\prod_{\substack{l=1 \\
l \neq k}}^{n S L} I\left[-\infty, T M P S L_{l}(t), f S L_{l}\right]\right\}\right. \\
& \left.\times\left\{1-\prod_{j=1}^{n W L} I\left[-\infty, T M P W L_{j}(t), f W L_{j}\right]\right\} \mathrm{d} T M P S L_{k}(t)\right) \\
& =\int_{t M I N}^{t M A X}\left(\sum_{k=1}^{n S L}\left\{f S L_{k}\left[T M P S L_{k}(t)\right]\right\}\left\{\prod_{\substack{l=1 \\
l \neq k}}^{n S L} I\left[-\infty, T M P S L_{l}(t), f S L_{l}\right]\right\}\left\{\mathrm{d} T M P S L_{k}(t) / \mathrm{d} t\right\}\right) \\
& \times\left(1-\prod_{j=1}^{n W L} I\left[-\infty, T M P W L_{j}(t), f W L_{j}\right]\right) \mathrm{d} t \\
& =\sum_{k=1}^{n S L} \int_{T M N S L_{k}}^{T M X S L_{k}}\left\{\left\{f S L_{k}\left(T_{S L}\right)\right\}\left\{\prod_{\substack{l=1 \\
l \neq k}}^{n S L} I\left[-\infty, T M P S L_{l}\left[T M P S L_{k}^{-1}\left(T_{S L}\right)\right], f S L_{l}\right]\right\}\right. \\
& \left.\times\left\{1-\prod_{j=1}^{n W L} I\left[-\infty, T M P W L_{j}\left[\operatorname{TMPSL}_{k}^{-1}\left(T_{S L}\right)\right], f W L_{j}\right]\right\}\right) \mathrm{d} T_{S L}
\end{aligned}
$$

with same notation as in Table 2. 
Table 9. Deviations from Decimal Results in Table 7 Observed in Determination of PLOAS with Sampling-Based Procedures and Samples of Size $10^{6}$

\begin{tabular}{|c|c|c|c|c|c|}
\hline \multicolumn{6}{|c|}{ Random Sampling } \\
\hline$n S L / n W L$ & 1 & 2 & 3 & 4 & 5 \\
\hline 1 & 0.00038 & 0.00008 & -0.00019 & -0.00004 & 0.00027 \\
\hline 2 & 0.00005 & -0.00030 & 0.00079 & 0.00010 & 0.00013 \\
\hline 3 & -0.00043 & 0.00027 & 0.00036 & -0.00022 & 0.00018 \\
\hline 4 & 0.00018 & 0.00029 & -0.00007 & 0.00004 & 0.00060 \\
\hline 5 & 0.00002 & 0.00044 & -0.00008 & 0.00099 & -0.00056 \\
\hline \multicolumn{6}{|c|}{ Importance Sampling: Uniform on $\left[c_{8}-4 c_{9}, c_{8}+4 c_{9}\right]=\left[271,342{ }^{\circ} \mathrm{C}\right]$} \\
\hline$n S L / n W L$ & 1 & 2 & 3 & 4 & 5 \\
\hline 1 & 0.00064 & -0.00089 & -0.00339 & -0.00339 & -0.00049 \\
\hline 2 & 0.00043 & -0.00271 & -0.00729 & -0.00756 & 0.00622 \\
\hline 3 & -0.00114 & -0.00347 & -0.00517 & 0.00624 & 0.01246 \\
\hline 4 & -0.00252 & -0.00350 & 0.00046 & 0.02284 & 0.02231 \\
\hline 5 & -0.00373 & 0.00351 & 0.01470 & 0.03057 & 0.05578 \\
\hline \multicolumn{6}{|c|}{ Importance Sampling: Normal with $\mu=c_{8}=310^{\circ}, \sigma=c_{9} / 2=4^{\circ} \mathrm{C}$} \\
\hline$n S L / n W L$ & 1 & 2 & 3 & 4 & 5 \\
\hline 1 & 0.00044 & -0.00020 & -0.00003 & 0.00007 & -0.00024 \\
\hline 2 & -0.00019 & -0.00031 & 0.00100 & 0.00002 & 0.00019 \\
\hline 3 & -0.00050 & 0.00047 & 0.00042 & 0.00004 & 0.00009 \\
\hline 4 & 0.00037 & 0.00029 & 0.00017 & -0.00006 & 0.00056 \\
\hline 5 & 0.00007 & 0.00066 & -0.00010 & 0.00091 & -0.00050 \\
\hline
\end{tabular}


This page intentionally left blank. 


\section{Problem 4: Failure of Any SL Before Failure of All WLS}

Verification Test Problem 4 is predicated on the assumption that loss of assured safety corresponds to failure of any SL before failure of all WLs and the additional assumption that the failures of the individual links are independent. With the indicated assumptions,

$$
\begin{aligned}
& \overline{p F}(t)=\sum_{k=1}^{n S L}\left(\int_{0}^{t}\left\{\prod_{\substack{l=1 \\
l \neq k}}^{n S L}\left[1-C D F_{S L, l}(\tau)\right]\right\}\left\{1-\prod_{j=1}^{n W L} C D F_{W L, j}(\tau)\right\} \mathrm{d} C D F_{S L, k}(\tau)\right)
\end{aligned}
$$

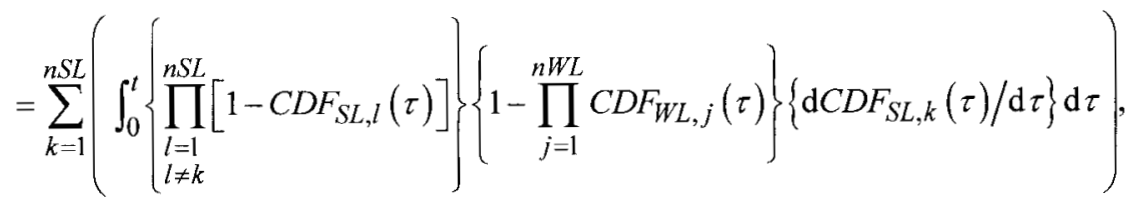

where the first integral is a Riemann-Stieltjes integral, the second integral is the corresponding Riemann integral, and $C D F_{W L, j}(\tau)$ and $C D F_{S L, j}(\tau)$ are defined the same as in Eq. (2.1).

The integrals defining $\overline{p F}(t)$ in Eq. (5.1) are obtained from the approximations

$$
\begin{aligned}
\overline{p F}(t) & \cong \sum_{k=1}^{n S L}\left(\sum_{i=1}^{n}\left\{\prod_{\substack{l=1 \\
l \neq k}}^{n S L}\left[1-C D F_{S L, l}\left(\tau_{i-1}\right)\right]\right\}\left\{1-\prod_{j=1}^{n W L} C D F_{W L, j}\left(\tau_{i-1}\right)\right\}_{2}\left\{C D F_{S L, k}\left(\tau_{i}\right)-C D F_{S L, k}\left(\tau_{i-1}\right)\right\}_{3}\right) \\
& =\sum_{k=1}^{n S L}\left\{\sum_{i=1}^{n}\left\{\prod_{\substack{l=1 \\
l \neq k}}^{n S L}\left[1-C D F_{S L, l}\left(\tau_{i-1}\right)\right]\right\}\left\{1-\prod_{j=1}^{n W L} C D F_{W L, j}\left(\tau_{i-1}\right)\right\}_{2}\left\{\frac{C D F_{S L, k}\left(\tau_{i}\right)-C D F_{S L, k}\left(\tau_{i-1}\right)}{\Delta \tau_{i}}\right\}_{4} \Delta \tau_{i}\right)
\end{aligned}
$$

for $0=\tau_{0}<\tau_{1}<\ldots<\tau_{n}=t$ and $\Delta \tau_{i}=\tau_{i}-\tau_{i-1}$. Specifically, $\{\sim\}_{1}$ is the probability that all SLs except SL $k$ have not failed before time $\tau_{i-1} ;\{\sim\}_{2}$ is the probability that at least one WL has not failed by time $\tau_{i-1} ;\{\sim\}_{3}$ is the probability that SL $k$ fails between $\tau_{i-1}$ and $\tau_{1}$; and $\{\sim\}_{4}$ is an approximation to $\mathrm{d} C D F_{S L, k}(\tau) / \mathrm{d} \tau$.

Similarly to previous results and with $p(\tau)$ defined the same as in Eq. (2.3), 


$$
\begin{aligned}
\overline{p F}(t) & =\sum_{k=1}^{n S L}\left(\int_{0}^{t}[1-p(\tau)]^{n S L-1}\left\{1-[p(\tau)]^{n W L}\right\} p^{\prime}(\tau) \mathrm{d} \tau\right) \\
& =(n S L) \int_{0}^{t}\left([1-p(\tau)]^{n S L-1}-[p(\tau)]^{n W L}[1-p(\tau)]^{n S L-1}\right) p^{\prime}(\tau) \mathrm{d} \tau \\
& =(n S L) \int_{p(0)}^{p(t)}\left([1-p]^{n S L-1}-p^{n W L}(1-p)^{n S L-1}\right) \mathrm{d} p .
\end{aligned}
$$

In turn,

$$
\begin{aligned}
\overline{p F}(\infty) & =\lim _{t \rightarrow \infty}(n S L) \int_{p(0)}^{p(t)}\left[(1-p)^{n S L-1}-p^{n W L}(1-p)^{n S L-1}\right] \mathrm{d} p \\
& =(n S L) \int_{0}^{1}\left[(1-p)^{n S L-1}-p^{n W L}(1-p)^{n S L-1}\right] \mathrm{d} p \\
& =(n S L)\left(\int_{0}^{1} p^{0}(1-p)^{n S L-1} \mathrm{~d} p-\int_{0}^{1} p^{n W L}(1-p)^{n S L-1} \mathrm{~d} p\right) \\
& =(n S L)([0 !(n S L-1) ! /(0+n S L) !]-[n W L !(n S L-1) ! /(n W L+n S L) !]) \\
& =(n S L)([1 / n S L]-[n W L !(n S L-1) ! /(n W L+n S L) !]) \\
& =1-[n W L ! n S L ! /(n W L+n S L) !],
\end{aligned}
$$

where the fourth equality follows from Eq. (2.6).

The relationship in Eq. (5.4) results in very simple and easily determined representations for PLOAS with different numbers of WLs and SLs when an analysis is carried out to the time at which all links have failed (Table 10). As an example, the results in Table 10 can be used to verify the representation for PLOAS shown of Table 11. The results of Table 11 are not included in the PLOAS representations given in Ref. [27] but can be derived in a similar manner. For this verification, all links are assumed to have the temperature curve and failure temperature density function defined in Eqs. (2.7) and (2.8).

The CPLOAS program was used to determine PLOAS with the indicated temperature curves and density functions for all combinations of WLs and SLs in Table 10. Further, calculations were carried out with each of the integration procedures implemented in CPLOAS (i.e., trapezoidal method, Simpson's method, simple random sampling, and importance sampling). The outcome was that the two quadrature methods produced results for all WL/SL combinations that agreed identically with the decimal representations for PLOAS in Table 10. The two sampling based methods produced results that were close to the decimal values in Table 10 but, as would be expected, were not in complete agreement (Table 12). 
Table 10. Values for PLOAS (i.e., $\overline{p F}(\infty)$ in Eq. (5.4)) for Different Numbers of WLs and SLs Predicated on the Assumptions that (i) Loss of Assured Safety Corresponds to Failure of Any SL Before Failure of All WLs, (ii) the Failures of the Individual Links are Independent, and (iii) All Links Have the Same Distribution for Failure Time

\begin{tabular}{|c|c|c|c|c|c|}
\hline \multicolumn{7}{|c|}{ Integer Ratio Representation } \\
\hline $\boldsymbol{n} \boldsymbol{S L} \boldsymbol{n} \boldsymbol{W} \boldsymbol{L}$ & $\mathbf{1}$ & $\mathbf{2}$ & $\mathbf{3}$ & $\mathbf{4}$ & $\mathbf{5}$ \\
\hline $\mathbf{1}$ & $1 / 2$ & $2 / 3$ & $3 / 4$ & $4 / 5$ & $5 / 6$ \\
\hline $\mathbf{2}$ & $2 / 3$ & $5 / 6$ & $9 / 10$ & $14 / 15$ & $20 / 21$ \\
\hline $\mathbf{3}$ & $3 / 4$ & $9 / 10$ & $19 / 20$ & $34 / 35$ & $55 / 56$ \\
\hline $\mathbf{4}$ & $4 / 5$ & $14 / 15$ & $34 / 35$ & $69 / 70$ & $125 / 126$ \\
\hline $\mathbf{5}$ & $5 / 6$ & $20 / 21$ & $55 / 56$ & $125 / 126$ & $251 / 252$ \\
\hline \multicolumn{7}{|c|}{ Decimal Fraction Representation } \\
\hline $\boldsymbol{n} \boldsymbol{S} \mathbf{n} \boldsymbol{W L}$ & $\mathbf{1}$ & $\mathbf{2}$ & $\mathbf{3}$ & $\mathbf{4}$ & $\mathbf{5}$ \\
\hline $\mathbf{1}$ & 0.50000 & 0.66667 & 0.75000 & 0.80000 & 0.83333 \\
\hline $\mathbf{2}$ & 0.66667 & 0.83333 & 0.90000 & 0.93333 & 0.95238 \\
\hline $\mathbf{3}$ & 0.75000 & 0.90000 & 0.95000 & 0.97143 & 0.98214 \\
\hline $\mathbf{4}$ & 0.80000 & 0.93333 & 0.97143 & 0.98571 & 0.99206 \\
\hline $\mathbf{5}$ & 0.83333 & 0.95238 & 0.98214 & 0.99206 & 0.99603 \\
\hline
\end{tabular}


Table 11. Representation of Value $p F$ for PLOAS Under Fire Conditions for WL/SL System With $n W L$ WLs, nSL SLs, and the Assumptions that (i) a Link Fails Instantly When it Reaches its Failure Temperature and (ii) Loss of Assured Safety Corresponds to Failure of Any SL Before Failure of all WLS

$$
\begin{aligned}
& p F=\sum_{k=1}^{n S L}\left(\int_{t M I N}^{t M A X}\left\{f S L_{k}\left[T M P S L_{k}(t)\right]\right\}\left\{\prod_{\substack{l=1 \\
l \neq k}}^{n S L} I\left[T M P S L_{l}(t), \infty, f S L_{l}\right]\right\}\right. \\
& \left.\times\left\{1-\prod_{j=1}^{n W L} I\left[-\infty, T M P W L_{j}(t), f W L_{j}\right]\right\} \mathrm{d} T M P S L_{k}(t)\right) \\
& =\int_{t M I N}^{t M A X}\left(\sum_{k=1}^{n S L}\left\{f S L_{k}\left[\operatorname{TMPSL}_{k}(t)\right]\right\}\left\{\prod_{\substack{l=1 \\
l \neq k}}^{n S L} I\left[T M P S L_{l}(t), \infty, f S L_{l}\right]\right\}\left\{\mathrm{d} T M P S L_{k}(t) / \mathrm{d} t\right\}\right) \\
& \times\left(1-\prod_{j=1}^{n W L} I\left[-\infty, T M P W L_{j}(t), f W L_{j}\right]\right) \mathrm{d} t \\
& =\sum_{k=1}^{n S L} \int_{T M N S L_{k}}^{T M X S L_{k}}\left\{\left\{f S L_{k}\left(T_{S L}\right)\right\}\left\{\prod_{\substack{l=1 \\
l \neq k}}^{n S L} I\left[T M P S L_{l}\left[\operatorname{TMPSL}_{k}^{-1}\left(T_{S L}\right)\right], \infty, f S L_{l}\right]\right\}\right. \\
& \left.\times\left\{1-\prod_{j=1}^{n W L} I\left[-\infty, T M P W L_{j}\left[\operatorname{TMPSL}_{k}^{-1}\left(T_{S L}\right)\right], f W L_{j}\right]\right\}\right) \mathrm{d} T_{S L}
\end{aligned}
$$

with same notation as in Table 2. 
Table 12. Deviations from Decimal Results in Table 10 Observed in Determination of PLOAS with Sampling-Based Procedures and Samples of Size $10^{6}$

\begin{tabular}{|c|c|c|c|c|c|}
\hline \multicolumn{6}{|c|}{ Random Sampling } \\
\hline$n S L / n W L$ & 1 & 2 & 3 & 4 & 5 \\
\hline 1 & 0.00038 & 0.00008 & -0.00019 & -0.00004 & -0.00027 \\
\hline 2 & 0.00077 & -0.00040 & 0.00015 & -0.00016 & 0.00009 \\
\hline 3 & -0.00007 & 0.00004 & 0.00007 & -0.00013 & 0.00009 \\
\hline 4 & -0.00039 & 0.00004 & 0.00003 & -0.00004 & 0.00000 \\
\hline 5 & 0.00012 & 0.00000 & 0.00011 & 0.00010 & 0.00001 \\
\hline \multicolumn{6}{|c|}{ Importance Sampling: Uniform on $\left[c_{8}-4 c_{9}, c_{8}+4 c_{9}\right]=\left[271,342^{\circ} \mathrm{C}\right]$} \\
\hline$n S L / n W L$ & 1 & 2 & 3 & 4 & 5 \\
\hline 1 & 0.00064 & -0.00089 & -0.00339 & -0.00339 & -0.00049 \\
\hline 2 & 0.00128 & -0.00002 & -0.00592 & -0.00382 & 0.00656 \\
\hline 3 & 0.00026 & 0.00156 & -0.00210 & -0.00470 & 0.00486 \\
\hline 4 & 0.00041 & -0.00115 & -0.00075 & 0.00125 & -0.00325 \\
\hline 5 & -0.00962 & 0.00260 & 0.00277 & 0.00264 & 0.00213 \\
\hline \multicolumn{6}{|c|}{ Importance Sampling: Normal with $\mu=c_{8}=310^{\circ}, \sigma=c_{9} / 2=4^{\circ} \mathrm{C}$} \\
\hline$n S L / n W L$ & 1 & 2 & 3 & 4 & 5 \\
\hline 1 & 0.00044 & -0.00020 & -0.00003 & 0.00007 & -0.00024 \\
\hline 2 & 0.00085 & -0.00037 & 0.00018 & -0.00028 & 0.00003 \\
\hline 3 & -0.00019 & 0.00011 & 0.00001 & -0.00017 & 0.00005 \\
\hline 4 & -0.00042 & -0.00013 & 0.00006 & -0.00009 & 0.00001 \\
\hline 5 & -0.00010 & 0.00015 & 0.00008 & 0.00002 & 0.00001 \\
\hline
\end{tabular}


This page intentionally left blank. 


\section{Alternate Derivations of Verification Test Problems}

The derivations of Verification Test Problems $1-4$ in Eqs. (2.5), (3.4), (4.4) and (5.4) are based on developing general, integration-based representations for PLOAS and then obtaining the resultant representations for PLOAS when all links are assumed to have the same distribution of failure time. This approach is appealing because of the direct connection between the general representations for PLOAS and the verification test problems. However, as shown in this section, the verification test problems can also be obtained from counting-based derivations involving set cardinality. These derivations are more succinct than the derivations leading to Eqs. (2.5), (3.4), (4.4) and (5.4) but lack the direct connection with the more general representations for PLOAS in Eqs. (2.1), (3.1), (4.1) and (5.1).

As before, a system involving $n W L$ WLs and $n S L$ SLs is under consideration. Further, the integer $j$ designates $\mathrm{WL} j$ for $j=1,2, \ldots, n W L$; the integer $n W L+k$ designates SL $k$ for $k=1,2, \ldots, n S L$; and

$$
n L=n W L+n S L
$$

is the total number of links. Then, a possible sequence of link failures can be represented by the vector

$$
\mathbf{n}=\left[n_{1}, n_{2}, \ldots, n_{n L}\right],
$$

where $n_{i}$ is the integer designator for the $i^{\text {th }}$ link to fail (i.e., link $n_{i}$ fails before link $n_{i+1}$ for $i=1,2, \ldots, n L-1$ ), and the set $S$ of all possible sequences of link failures is given by

$$
S=\left\{\mathbf{n}: \mathbf{n}=\left[n_{1}, n_{2}, \ldots, n_{n L}\right], n_{i} \text { is an integer between } 1 \text { and } n L, \text { and } n_{i} \neq n_{j} \text { for } i \neq j\right\} .
$$

The cardinality $C(S)$ of $S$ is

$$
C(S)=n L !=(n W L+n S L) !
$$

and corresponds to the total number of orderings of WL/SL failures. As shown in Sects. $6.1-6.4$, the individual verification test problems can be derived using the cardinality $C(S)$ of $S$. Further, as shown in Sect. 6.5 , the test problems can also be derived from the hypergeometric probability distribution.

\subsection{Problem 1: Failure of All SLs before Failure of Any WL}

The set $S_{1}$ of all possible sequences of link failures that meet the criterion for loss of assured safety associated with Verification Test Problem 1 (i.e., failure of all SLs before failure of any WL) is

$$
S_{1}=\left\{\mathbf{n}: \mathbf{n}=\left[n_{1}, n_{2}, \ldots, n_{n L}\right] \in S \text { and } n W L+1 \leq n_{i} \leq n L \text { for } i=1,2, \ldots, n S L\right\}
$$

In turn, the cardinality of $C\left(S_{1}\right)$ of $S_{1}$ is 


$$
C\left(S_{1}\right)=n W L ! n S L !
$$

As a result, PLOAS is given by

$$
\overline{p F}(\infty)=C\left(S_{1}\right) / C(S)=n W L ! n S L !(n W L+n S L) !
$$

which is the same as the representation in Eq. (2.5).

\subsection{Problem 2: Failure of Any SL before Failure of Any WL}

The set $S_{2}$ of all possible sequences of link failures that meet the criterion for loss of assured safety associated with Verification Test Problem 2 (i.e., failure of any SL before failure of any WL) is

$$
S_{2}=\left\{\mathbf{n}: \mathbf{n}=\left[n_{1}, n_{2}, \ldots, n_{n L}\right] \in S \text { and } n W L+1 \leq n_{1} \leq n L\right\}
$$

In turn, the cardinality $C\left(S_{2}\right)$ of $S_{2}$ is

$$
C\left(S_{2}\right)=n S L(n W L+n S L-1) !
$$

As a result, PLOAS is given by

$$
\begin{aligned}
\overline{p F}(\infty) & =C\left(S_{2}\right) / C(S) \\
& =n S L(n W L+n S L-1) ! /(n W L+n S L) ! \\
& =n S L /(n W L+n S L),
\end{aligned}
$$

which is the same as the representation in Eq. (3.4).

\subsection{Problem 3: Failure of All SLs before Failure of All WLs}

The set $S_{3}$ of all possible sequences of link failures that meet the criterion for loss of assured safety associated with Verification Test Problem 3 (i.e., failure of all SLs before failure of all WLs) is

$$
S_{3}=\left\{\mathbf{n}: \mathbf{n}=\left[n_{1}, n_{2}, \ldots, n_{n L}\right] \in S \text { and } \mathbf{1} \leq n_{n L} \leq n W L\right\}
$$

In turn, the cardinality $C\left(S_{3}\right)$ of $S_{3}$ is

$$
C\left(S_{3}\right)=(n W L+n S L-1) ! n W L .
$$

As a result, PLOAS is given by 


$$
\begin{aligned}
\overline{p F}(\infty) & =C\left(S_{3}\right) / C(S) \\
& =(n W L+n S L-1) ! n W L /(n W L+n S L) ! \\
& =n W L /(n W L+n S L),
\end{aligned}
$$

which is the same as the representation in Eq. (4.4).

\subsection{Problem 4: Failure of Any SL before Failure of All WLs}

The complement $S_{4}^{c}$ of the set $S_{4}$ of all possible sequences of link failures that meet the criterion for loss of assured safety associated with Verification Test Problem 4 (i.e., failure of any SL before failure of all WLs) is

$$
S_{4}^{\mathcal{C}}=\left\{\mathbf{n}: \mathbf{n}=\left[n_{1}, n_{2}, \ldots, n_{n L}\right] \in S \text { and } 1 \leq n_{i} \leq n W L \text { for } i=1,2, \ldots, n W L\right\}
$$

In turn, the cardinalities $C\left(S_{4}^{c}\right)$ and $C\left(S_{4}\right)$ of $S_{4}^{c}$ and $S_{4}$ are

$$
C\left(S_{4}^{c}\right)=n W L ! n S L !
$$

and

$$
C\left(S_{4}\right)=C(S)-C\left(S_{4}^{c}\right)=(n W L+n S L) !-n W L ! n S L !
$$

As a result, PLOAS is given by

$$
\begin{aligned}
\overline{p F}(\infty) & =C\left(S_{4}\right) / C(S) \\
& =[(n W L+n S L) !-n W L ! n S L !] /(n W L+n S L) ! \\
& =1-[n W L ! n S L ! /(n W L+n S L) !]
\end{aligned}
$$

which is the same as the representation in Eq. (5.4).

\subsection{Derivation of Verification Test Problems Based on Hypergeometric Probability Distribution}

The four verification test problems can also be derived from the hypergeometric probability distribution (Sect. 4.2, Ref. [37]). This distribution characterizes the probabilities of the different possible outcomes of randomly drawing $n S$ objects without replacement from a collection of two different types of objects. Specifically, if the collection of objects contains $n O 1$ objects of Type 1 (e.g., white balls) and $n O 2$ objects of Type 2 (e.g., black balls), then the probability $p(n O)$ of drawing a sample of size $n S$ that contains exactly $n O$ objects of Type 1 is 


$$
p(n O)=\left(\begin{array}{c}
n O 1 \\
n O
\end{array}\right)\left(\begin{array}{c}
n O 2 \\
n S-n O
\end{array}\right) /\left(\begin{array}{c}
n O 1+n O 2 \\
n S
\end{array}\right)
$$

The basic assumption underlying the derivation of $p(n O)$ is that each object in the collection is equally likely to be picked in the generation of the indicated sample of size $n S$.

Verification Test Problem 1 is predicated on the assumption that loss of assured safety corresponds to the fâilure of all SLs before the failure of any WL. In this case, $\overline{p F}(\infty)$ is the probability that all SLs fail before any WL fails and conceptually corresponds to drawing a sample of size $n S L$ from the collection of $n W L+n S L$ links that contains only SLs. As a result, and with $n O 1=n S L, n O 2=n W L, n O=n S L$ and $n S=n S L$ in the notation of Eq. (6.18), $\overline{p F}(\infty)$ is given by

$$
\begin{aligned}
\overline{p F}(\infty) & =p(n S L) \\
& =\left(\begin{array}{c}
n S L \\
n S L
\end{array}\right)\left(\begin{array}{c}
n W L \\
n S L-n S L
\end{array}\right) /\left(\begin{array}{c}
n S L+n W L \\
n S L
\end{array}\right) \\
& =n W L ! n S L ! /(n W L+n S L) !
\end{aligned}
$$

which is the same as the representation for $\overline{p F}(\infty)$ in Eqs. (2.5) and (6.7).

Verification Test Problem 2 is predicated on the assumption that loss of assured safety corresponds to the failure of any SL before any WL. In this case, $\overline{p F}(\infty)$ is the probability that the first link to fail is a SL and conceptually corresponds to drawing a sample of size 1 from the collection of $n W L+n S L$ links that contain a SL. As a result, and with $n O 1=n S L, n O 2=n W L, n O=1$ and $n S=1$ in the notation of Eq. (6.18), $\overline{p F}(\infty)$ is given by

$$
\begin{aligned}
\overline{p F}(\infty) & =p(1) \\
& =\left(\begin{array}{c}
n S L \\
1
\end{array}\right)\left(\begin{array}{c}
n W L \\
1-1
\end{array}\right) /\left(\begin{array}{c}
n S L+n W L \\
1
\end{array}\right) \\
& =n S L /(n W L+n S L),
\end{aligned}
$$

which is the same as the representation for $\overline{p F}(\infty)$ in Eqs. (3.4) and (6.10).

Verification Test Problem 3 is predicated on the assumption that loss of assured safety corresponds to failure of all SLs before failure of all WLs. In this case, $\overline{p F}(\infty)$ is the probability that the last link to fail is a WL and conceptually corresponds to drawing a sample of size 1 from the collection of $n W L+n S L$ links that contains a WL. As a result, and with $n O 1=n W L, n O 2=n S L, n O=1$ and $n S=1$ in the context of Eq. (6.18), $\overline{p F}(\infty)$ is given by 


$$
\begin{aligned}
p F(\infty) & =p(1) \\
& =\left(\begin{array}{c}
n W L \\
1
\end{array}\right)\left(\begin{array}{l}
n S L \\
1-1
\end{array}\right) /\left(\begin{array}{c}
n W L+n S L \\
1
\end{array}\right) \\
& =n W L /(n W L+n S L),
\end{aligned}
$$

which is the same as the representation for $\overline{p F}(\infty)$ in Eqs. (4.4) and (6.13).

Verification Test Problem 4 is predicated on the assumption that loss of assured safety corresponds to the failure of any SL before the failure of all WLs. In this case, $\overline{p F}(\infty)$ is the probability that at least one SL fails before all WLs fail and conceptually corresponds to not drawing a sample of size $n W L$ from the collection of $n W L+n S L$ links that contains only WLs. As a result, and with $n O 1=n W L, n O 2=n S L, n O=n W L$ and $n S=n W L$ in the context of Eq. (6.18), $\overline{p F}(\infty)$ is given by

$$
\begin{aligned}
\overline{p F}(\infty) & =1-p(n W L) \\
& =1-\left(\begin{array}{l}
n W L \\
n W L
\end{array}\right)\left(\begin{array}{c}
n S L \\
n W L-n W L
\end{array}\right) /\left(\begin{array}{c}
n W L+n S L \\
n W L
\end{array}\right) \\
& =1-[n W L ! n S L ! /(n W L+n S L) !]
\end{aligned}
$$

which is the same as the representation for $\overline{p F}(\infty)$ in Eqs. (5.4) and (6.17). 
This page intentionally left blank. 


\section{Discussion}

The determination of PLOAS in a temperature dependent system with multiple WLs and SLs involves a conceptually and computationally complex calculation. At the core of this calculation is the evaluation of an iterated integral, where the number of individual integrals is equal to the total number of WLs and SLs. As a result of its complexity, this integral must be evaluated numerically, which makes a direct verification of its evaluation (e.g., by comparing with hand calculations) infeasible. Yet, without adequate verification, little confidence can be placed in the results of a calculation of this complexity.

One approach to verification is to compare results obtained with different numerical solution procedures. As an example, the PLOAS development in Ref. [27] describes and illustrates two quadrature-based approaches (i.e., trapezoidal method and Simpson's method) and two sampling-based methods (i.e., simple random sampling and importance sampling). Agreement in results when the same problem is solved with different numerical procedures helps provide assurance that the obtained results are correct. This is particularly true when the numerical methods are different in their mode of operation. For example, this is the case when results obtained with quadrature-based methods and sampling-based methods are compared as these methods are quite different in their implementation and mode of operation. However, agreement of results obtained with different numerical procedures does not rule out some underlying error in the derivation of the problem.

An approach to verification that has the potential to identify errors in the development of the problem is to compare results obtained in two independent derivations and numerical implementations of the same problem (e.g., by two different individuals or two different teams of individuals). Such a comparison for PLOAS calculations is reported in Sect. 7.2 of Ref. [11], with this comparison resulting in the identification of an error in one of the calculations. Although this approach to verification is appealing, in practice it is not very useful as the resources required for two complete, independent developments of a problem are unlikely to be available in most real analyses.

Another approach to verification is to use test problems with known solutions. Then, failure to agree with the known solution indicates that there is an error in the conceptual development or numerical implementation of the problem. In contrast, agreement with the known solution helps provide assurance, but not complete proof, that the problem is being correctly implemented and solved. As an example, an appropriately chosen test problem helped identify the error found in the verification of PLOAS calculations described in Sect. 7.2 of Ref. [11]. Well-chosen test problems are very effective in the verification of complex calculations because they can force a full exercise of the numerics associated with a problem while providing a known solution with which to compare calculated results.

This presentation describes four verification test problems for checking the conceptual development and computational implementation of calculations to determine PLOAS in temperature-dependent systems with multiple WLs and SLs. The problems are designed to test results obtained with the following definitions of loss of assured 
safety: (i) Failure of all SLs before failure of any WL, (ii) Failure of any SL before failure of any WL, (iii) Failure of all SLs before failure of all WLs, and (iv) Failure of any SL before failure of all WLs. The test problems are based on assuming the same failure properties for all links, which results in problems that have the desirable properties of fully exercising the numerical integration procedures required in the evaluation of PLOAS and also possessing simple algebraic representations for PLOAS that can be used for verification of the analysis. This duality of fully exercising the numerics involved in calculating PLOAS while still possessing known solutions makes these problems very effective tests for use in the verification of PLOAS calculations.

Although not discussed in this presentation, sampling-based sensitivity analysis procedures provide an additional tool for use in analysis verification. ${ }^{38-42}$ With this approach to analysis verification, a sampling-based mapping between analysis inputs and analysis results is developed. Then, a variety of sensitivity analysis procedures can be used to determine the effects of analysis inputs on analysis results. The presence of anomalous or clearly erroneous relationships indicates errors in the analysis. In contrast, the presence of appropriate and explainable relationships between analysis inputs and analysis results provides a strong indication, but not an absolute proof, that the analysis is correctly implemented.

No single verification procedure can ever establish that a complex analysis has been developed and implemented correctly. Therefore, it is recommended that analysis verification be carried out with multiple procedures whenever possible. 


\section{References}

1. Trucano, T.G., L.P. Swiler, T. Igusa, W.L. Oberkampf, and M. Pilch. 2006. "Calibration, Validation, and Sensitivity Analysis: What's What," Reliability Engineering and System Safety, to appear.

2. Babuska, I. and J.T. Oden. 2004. "Verification and Validation in Computational Engineering and Science: Basic Concepts," Computer Methods in Applied Mechanics and Engineering. Vol. 193, no. 36-38, pp. 40574066 .

3. Oberkampf, W.L., T.G. Trucano, and C. Hirsch. 2004. "Verification, Validation, and Predictive Capability in Computational Engineering and Physics," Applied Mechanics Review. Vol. 57, no. 5, pp. 345-384.

4. Roache, P.J. 2004. "Building PDE Codes to be Verifiable and Validatable," Computing in Science \& Engineering. Vol. 6, no. 5, pp. 30-38.

5. Knupp, P. and K. Salari. 2002. Verification of Computer Codes in Computational Science and Engineering. Boca Raton, FL: Chapman and Hall/CRC.

6. Oberkampf, W.L., S.M. DeLand, B.M. Rutherford, K.V. Diegert, and K.F. Alvin. 2002. "Error and Uncertainty in Modeling and Simulation," Reliability Engineering and System Safety. Vol. 75, no. 3, pp. 333-357.

7. Oberkampf, W.L. and T.G. Trucano. 2002. "Verification and Validation in Computational Fluid Dynamics," Progress in Aerospace Sciences. Vol. 38, no. 3, pp. 209-272.

8. American Institute of Aeronautics and Astronautics. 1998. AIAA Guide for the Verification and Validation of Computational Fluid Dynamics Simulations, AIAA G-077-1998. Reston, VA: American Institute of Aeronautics and Astronautics.

9. Roache, P.J. 1998. Verification and Validation in Computational Science and Engineering. Albuquerque, NM: Hermosa Publishers.

10. Roache, P.J. 1997. "Quantification of Uncertainty in Computational Fluid Dynamics," Annual Review of Fluid Mechanics. Vol. 29, pp. 123-160.

11. Helton, J.C., J.D. Johnson, and W.L. Oberkampf. 2004. Probability of Loss of Assured Safety in Temperature Dependent Systems with Multiple Weak and Strong Links, SAND2004-5216. Albuquerque, NM: Sandia National Laboratories.

12. Crowder, M.J. 2001. Classical Competing Risks. Boca Raton, FL: Chapman \& Hall/CRC. 
13. David, H.A. and M.L. Moeschberger. 1978. The Theory of Competing Risks. London: Griffin.

14. Seal, H.L. 1977. "Studies in the History of Probability and Statistics, XXXV. Multiple Decrements or Competing Risks," Biometrika. Vol. 64, pp. 429-439.

15. Gail, M. 1975. "Review and Critique of Some Models Used in Competing Risk Analysis," Biometrics. Vol. 31, no. 1, pp. 209-222.

16. Bartle, R.G. 1975. The Elements of Real Analysis (2nd Ed.). New York, NY: John Wiley \& Sons.

17. Helton, J.C. 1997. "Uncertainty and Sensitivity Analysis in the Presence of Stochastic and Subjective Uncertainty," Journal of Statistical Computation and Simulation. Vol. 57, no. 1-4, pp. 3-76.

18. Helton, J.C. and D.E. Burmaster. 1996. "Guest Editorial: Treatment of Aleatory and Epistemic Uncertainty in Performance Assessments for Complex Systems," Reliability Engineering and System Safety. Vol. 54, no. 2-3, pp. 91-94.

19. Paté-Cornell, M.E. 1996. "Uncertainties in Risk Analysis: Six Levels of Treatment," Reliability Engineering and System Safety. Vol. 54, no. 2-3, pp. 95-111.

20. Winkler, R.L. 1996. "Uncertainty in Probabilistic Risk Assessment," Reliability Engineering and System Safety. Vol. 54, no. 2-3, pp. 127-132.

21. Hoffman, F.O. and J.S. Hammonds. 1994. "Propagation of Uncertainty in Risk Assessments: The Need to Distinguish Between Uncertainty Due to Lack of Knowledge and Uncertainty Due to Variability," Risk Analysis. Vol. 14, no. 5, pp. 707-712.

22. Helton, J.C. 1994. "Treatment of Uncertainty in Performance Assessments for Complex Systems," Risk Analysis. Vol. 14, no. 4, pp. 483-511.

23. Apostolakis, G. 1990. "The Concept of Probability in Safety Assessments of Technological Systems," Science. Vol. 250, no. 4986, pp. 1359-1364.

24. Haan, C.T. 1989. "Parametric Uncertainty in Hydrologic Modeling," Transactions of the ASAE. Vol. 32, no. 1, pp. 137-146.

25. Parry, G.W. and P.W. Winter. 1981. "Characterization and Evaluation of Uncertainty in Probabilistic Risk Analysis," Nuclear Safety. Vol. 22, no. 1, pp. 28-42. 
26. D. Zwillinger (Ed.). 1996. CRC Standard Mathematical Tables and Formulae. 30th ed. Boca Raton, FL: CRC Press.

27. Helton, J.C., J.D. Johnson, and W.L. Oberkampf. 2006. "Probability of Loss of Assured Safety in Temperature Dependent Systems with Multiple Weak and Strong Links," Reliability Engineering and System Safety. Vol. 91, no. 3, pp. 320-348.

28. Evans, M. and T. Swartz. 2000. Approximating Integrals via Monte Carlo and Deterministic Methods. Oxford, New York: Oxford University Press.

29. Hurtado, J.E. and A.H. Barbat. 1998. "Monte Carlo Techniques in Computational Stochastic Mechanics," Archives of Computational Methods in Engineering. Vol. 5, no. 1, pp. 3-330.

30. Nicola, V.F., P. Shahabuddin, and M.K. Nakayama. 2001. "Techniques for Fast Simulation of Models of Highly Dependable Systems," IEEE Transactions on Reliability. Vol. 50, no. 3, pp. 246-264.

31. Owen, A. and Y. Zhou. 2000. "Safe and Effective Importance Sampling," Journal of American Statistical Association. Vol. 95, no. 449, pp. 135-143.

32. Heidelberger, P. 1995. "Fast Simulation of Rare Events in Queueing and Reliability Models," ACM Transactions on Modeling and Computer Simulation. Vol. 5, no. 1, pp. 43-85.

33. Shahabuddin, P. 1994. "Importance Sampling for the Simulation of Highly Reliable Markovian Systems," Management Science. Vol. 40, no. 3, pp. 333-352.

34. Goyal, A., P. Shahabuddin, P. Heidelberger, V.F. Nicola, and P.W. Glynn. 1992. "A Unified Framework for Simulating Markovian Models of Highly Dependable Systems," IEEE Transactions on Computers. Vol. 41, no. 1 , pp. 36-51.

35. Melchers, R.E. 1990. "Search-Based Importance Sampling," Structural Safety. Vol. 9, no. 2, pp. 117-128.

36. Glynn, P.W. and D.L. Iglehart. 1989. "Importance Sampling for Stochastic Simulations," Management Science. Vol. 35, no. 11, pp. 1367-1392.

37. Hahn, G.J. and S.S. Shapiro. 1967. Statistical Models in Engineering. New York: John Wiley \& Sons.

38. Helton, J.C., J.D. Johnson, C.J. Sallaberry, and C.B. Storlie. 2006. "Survey of Sampling-Based Methods for Uncertainty and Sensitivity Analysis," Reliability Engineering and System Safety, to appear. 
39. Helton, J.C. and F.J. Davis. 2003. "Latin Hypercube Sampling and the Propagation of Uncertainty in Analyses of Complex Systems," Reliability Engineering and System Safety. Vol. 81, no. 1, pp. 23-69.

40. Helton, J.C. and F.J. Davis. 2002. "Illustration of Sampling-Based Methods for Uncertainty and Sensitivity Analysis," Risk Analysis. Vol. 22, no. 3, pp. 591-622.

41. Helton, J.C. and F.J. Davis. 2000. "Sampling-Based Methods," Sensitivity Analysis. Ed. A. Saltelli, K. Chan, and E.M. Scott. New York, NY: Wiley. pp. 101-153.

42. Kleijnen, J.P.C. and J.C. Helton. 1999. "Statistical Analyses of Scatterplots to Identify Important Factors in Large-Scale Simulations, 1: Review and Comparison of Techniques," Reliability Engineering and System Safety. Vol. 65, no. 2, pp. 147-185. 


\section{DISTRIBUTION}

\section{External Distribution}

Scott Ferson

Applied Biomathematics

100 North Country Road

Setauket, New York 11733-1345

Sankaran Mahadevan

Dept. of Civil \& Environmental Engineering

Vanderbilt University

Box 6077, Station B

Nashville, TN 37235

Prof. Joseph Powers

Dept. of Aerospace and Mechanical Engr.

University of Notre Dame

Notre Dame, IN 46556-5637

Patrick J. Roache

1215 Apache Drive

Socorro, NM 87801

Prof. Chris Roy

Dept. of Aerospace Engineering

211 Aerospace Engineering Bldg.

Auburn University, AL 36849-5338

Simone Youngblood (2)

DOD/DMSO

Technical Director for VV\&A

1901 N. Beauregard St., Suite 504

Alexandria, VA 22311

\section{Department of Energy Laboratories}

Department of Energy (5)

Attn: Kevin Greenaugh, NA-115

D. Kusnezov, NA-114

Jamileh Soudah, NA-114

K. Sturgess, NA-115

J. Van Fleet, NA-113

Forrestal Building

1000 Independence Ave., SW

Washington, DC 20585
Los Alamos National Laboratory (13)

Mail Station 5000

P.O. Box 1663

Los Alamos, NM 87545

Mark C. Anderson, MS T080

Jerry S. Brock, MS F663

Scott Doebling, MS T080

Francois Hemez, MS F699

David Higdon, MS F600

Cliff Joslyn, MS B265

James Kamm, MS D413

Jonathan Lucero, MS T001

James Peery, MS F652

William Rider, MS D413

Kari Sentz, MS F600

David Sharp, MS B213

Alyson G. Wilson, MS F600

Lawrence Livermore National Laboratory (8)

7000 East Ave.

P.O. Box 808

Livermore, CA 94550

John Bolstad, MS L-023

Frank Graziani, MS L-095

Richard Klein, MS L-023

Roger Logan, MS L-125

J. F. McEnerney, MS L-023

Kambiz Salari, MS L-228

Joe Sefcik, MS L-160

Carol Woodward, MS L-561

\section{Sandia Internal Distribution}

$\begin{array}{lll}\text { MS 1110 } & 1410 & \text { D. E. Womble } \\ \text { MS 0370 } & 1411 & \text { S. A. Mitchell } \\ \text { MS 0370 } & 1411 & \text { M. S. Eldred } \\ \text { MS 1111 } & 1411 & \text { P. Knupp } \\ \text { MS 0370 } & 1411 & \text { L. P. Swiler } \\ \text { MS 0370 } & 1411 & \text { T. G. Trucano } \\ \text { MS 0384 } & 1500 & \text { A.C. Ratzel } \\ \text { MS 0824 } & 1500 & \text { T.Y. Chu } \\ \text { MS 0836 } & 1516 & \text { D. Dobranich } \\ \text { MS 0847 } & 1524 & \text { J. M. Redmond } \\ \text { MS 0824 } & 1530 & \text { A.L. Thornton } \\ \text { MS 1135 } & 1532 & \text { S. R. Tieszen } \\ \text { MS 0828 } & 1533 & \text { M. Pilch } \\ \text { MS 0828 } & 1533 & \text { A. R. Black } \\ \text { MS 0828 } & 1533 & \text { K. J. Dowding } \\ \text { MS 0828 } & 1533 & \text { A. A. Giunta } \\ \text { MS 0779 } & 1533 & \text { J. C. Helton } \\ \text { MS 0828 } & 1533 & \text { W. L. Oberkampf } \\ \text { MS 0557 } & 1533 & \text { T. L. Paez } \\ \text { MS 0828 } & 1533 & \text { V. J. Romero }\end{array}$




$\begin{array}{llll}1 & \text { MS 0828 } & 1533 & \text { A. Urbina } \\ 1 & \text { MS 0384 } & 1540 & \text { H. S. Morgan } \\ 1 & \text { MS 0380 } & 1542 & \text { K. F. Alvin } \\ 1 & \text { MS 0382 } & 1543 & \text { J. R. Stewart } \\ 1 & \text { MS 0139 } & 1900 & \text { A. Hale } \\ 1 & \text { MS 0139 } & 1902 & \text { P. Yarrington } \\ 1 & \text { MS 0139 } & 1904 & \text { R. K. Thomas } \\ 1 & \text { MS 0437 } & 2100 & \text { B. C. Walker } \\ 1 & \text { MS 0453 } & 2110 & \text { L. S. Walker } \\ 1 & \text { MS 0427 } & 2118 & \text { R. A. Paulsen } \\ 1 & \text { MS 0427 } & 2118 & \text { S. E. Klenke } \\ 1 & \text { MS 0453 } & 2120 & \text { M. R. Sjulin } \\ 1 & \text { MS 0482 } & 2123 & \text { E. R. Hoover } \\ 1 & \text { MS 0453 } & 2130 & \text { M. A. Rosenthal } \\ 1 & \text { MS 0481 } & 2132 & \text { S. G. Barnhart } \\ 1 & \text { MS 0481 } & 2137 & \text { J. F. Nagel }\end{array}$

$\begin{array}{llll}1 & \text { MS 9159 } & 8962 & \text { P. D. Hough } \\ 1 & \text { MS 9159 } & 8962 & \text { M. L. Martinez-Canales } \\ 1 & \text { MS 0428 } & 12300 & \text { C. L. Knapp } \\ 1 & \text { MS 0428 } & 12330 & \text { T. R. Jones } \\ 1 & \text { MS 0434 } & 12334 & \text { B. M. Mickelsen } \\ 1 & \text { MS 0830 } & 12335 & \text { K. V. Diegert } \\ 1 & \text { MS 0829 } & 12337 & \text { J. M. Sjulin } \\ 1 & \text { MS 0428 } & 12340 & \text { V. J. Johnson } \\ 1 & \text { MS 0428 } & 12341 & \text { N. J. DeReu } \\ 1 & \text { MS 0405 } & 12346 & \text { R. Kreutzeld } \\ 1 & \text { MS 0405 } & 12346 & \text { S. E. Camp } \\ 1 & \text { MS 0405 } & 12347 & \text { L-J Shyr } \\ 1 & \text { MS 0405 } & 12347 & \text { T. D. Brown } \\ 2 & \text { MS 9960 } & 8945-1 & \text { Central Technical Files } \\ 2 & \text { MS 0899 } & 04536 & \text { Technical Library }\end{array}$

